\title{
A non-grey analytical model for irradiated atmospheres ${ }^{\star}$
}

\section{Derivation}

\author{
Vivien Parmentier ${ }^{1,2}$ and Tristan Guillot ${ }^{1,2}$ \\ ${ }^{1}$ Laboratoire Lagrange, UMR7293, Université de Nice Sophia-Antipolis, CNRS, Observatoire de la Côte d'Azur, 06300 Nice, France \\ e-mail: vivien.parmentier@oca.eu \\ 2 Department of Astronomy and Astrophysics, University of California, Santa Cruz, CA 95064, USA
}

Received 22 July 2013 / Accepted 25 November 2013

\begin{abstract}
Context. Semi-grey atmospheric models (with one opacity for the visible and one opacity for the infrared) are useful for understanding the global structure of irradiated atmospheres, their dynamics, and the interior structure and evolution of planets, brown dwarfs, and stars. When compared to direct numerical radiative transfer calculations for irradiated exoplanets, however, these models systematically overestimate the temperatures at low optical depths, independently of the opacity parameters.

Aims. We investigate why semi-grey models fail at low optical depths and provide a more accurate approximation to the atmospheric structure by accounting for the variable opacity in the infrared.

Methods. Using the Eddington approximation, we derive an analytical model to account for lines and/or bands in the infrared. Four parameters (instead of two for the semi-grey models) are used: a visible opacity $\left(\kappa_{\mathrm{v}}\right)$, two infrared opacities, $\left(\kappa_{1}\right.$ and $\left.\kappa_{2}\right)$, and $\beta$ (the fraction of the energy in the beam with opacities $\kappa_{1}$ ). We consider that the atmosphere receives an incident irradiation in the visible with an effective temperature $T_{\text {irr }}$ and at an angle $\mu_{*}$, and that it is heated from below with an effective temperature $T_{\text {int }}$.

Results. Our non-grey, irradiated line model is found to provide a range of temperatures that is consistent with that obtained by numerical calculations. We find that if the stellar flux is absorbed at optical depth larger than $\tau_{\lim }=\left(\kappa_{\mathrm{R}} / \kappa_{1} \kappa_{2}\right)\left(\kappa_{\mathrm{R}} \kappa_{\mathrm{P}} / 3\right)^{1 / 2}$, it is mainly transported by the channel of lowest opacity whereas if it is absorbed at $\tau \gtrsim \tau_{\lim }$ it is mainly transported by the channel of highest opacity, independently of the spectral width of those channels. For low values of $\beta$ (expected when lines are dominant), we find that the non-grey effects significantly cool the upper atmosphere. However, for $\beta \gtrsim 1 / 2$ (appropriate in the presence of bands with a wavelength-dependence smaller than or comparable to the width of the Planck function), we find that the temperature structure is affected down to infrared optical depths unity and deeper as a result of the so-called blanketing effect.

Conclusions. The expressions that we derive can be used to provide a proper functional form for algorithms that invert the atmospheric properties from spectral information. Because a full atmospheric structure can be calculated directly, these expressions should be useful for simulations of the dynamics of these atmospheres and of the thermal evolution of the planets. Finally, they should be used to test full radiative transfer models and to improve their convergence.
\end{abstract}

Key words. radiative transfer - planets and satellites: atmospheres - stars: atmospheres - planetary systems

\section{Introduction}

The discovery of numerous star-planet systems and the possibility of characterizing the planets' atmospheric properties has led to a great many publications using radiative transfer calculations, often taken "off-the-shelf" from numerical models. Given the infinite amount of possible compositions for mostly unknown exoplanetary atmospheres, it is highly valuable to be able to perform very fast calculations and also to understand what determines the thermal structure of an irradiated atmosphere.

Analytical radiative transfer solutions for atmospheres have been calculated with a variety of assumptions and in different contexts (e.g. Eddington 1916; Chandrasekhar 1935, 1960; King 1956; Matsui \& Abe 1986; Weaver \& Ramanathan 1995; Pujol \& North 2003; Chevallier et al. 2007; Shaviv et al. 2011). However, the discovery of super-Earths, giant exoplanets, brown dwarfs, and low-mass stars close to a source of intense radiation has prompted the need for solutions that account for both

* A FORTRAN implementation of the analytical model is available at the CDS via anonymous ftp to cdsarc.u-strasbg. fr

$(130.79 .128 .5)$ or via

http: //cdsarc.u-strasbg.fr/viz-bin/qcat?]/A+A/562/A133 an outside and an inside radiation field, and properly link low and high optical depths levels. Hubeny et al. (2003), Rutily et al. (2008), Hansen (2008), Guillot (2010), Robinson \& Catling (2012), and Heng et al. (2012) provide these solutions in the framework of a semi-grey model, with one opacity for the incoming irradiation (generally mostly at visible wavelengths), and one opacity for the thermal radiation field (generally mostly at infrared wavelengths). These approximations have been used in hydrodynamical models of planetary atmospheres (e.g. Heng et al. 2011; Rauscher \& Menou 2013), planetary evolution models (e.g. Miller-Ricci \& Fortney 2010; Guillot \& Havel 2011; Budaj et al. 2012), planet synthesis models (Mordasini et al. 2012a,b), retrieval methods (Line et al. 2012), and a variety of other applications.

As shown in Fig. 1 for an atmosphere irradiated from above with a flux $\sigma T_{\text {irr }}^{4}$ and heated from below with a flux $\sigma T_{\text {int }}^{4}$, while semi-grey models provide solutions that are well-behaved when compared to full numerical solutions at optical depths larger than about unity, the temperatures at low-optical depths appear to be systematically hotter than in the numerical solutions. Most importantly, this occurs regardless of the choice of the two parameters of the problem, i.e. the thermal (infrared) opacity $\kappa_{\mathrm{th}}$ 


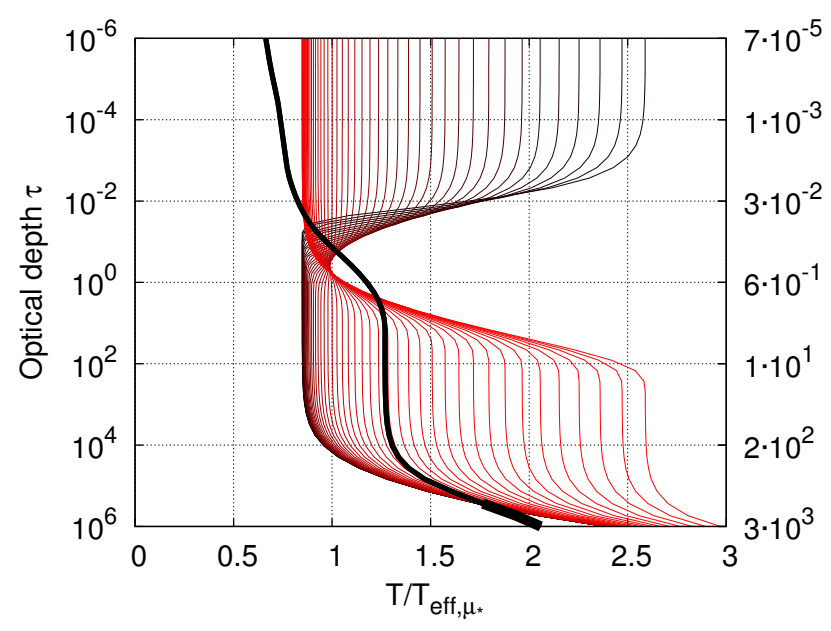

Fig. 1. Optical depth vs. atmospheric temperature in units of the effective temperature. A numerical solution obtained from Fortney et al. (2008) (thick black line) is compared to the semi-grey analytical solutions of Guillot (2010) for values of the greenhouse factor $\gamma_{\mathrm{v}}^{-1}$ ranging from 0.01 to 100 (black to red lines). Low values of $\gamma_{\mathrm{v}}$ are redder. We used $\mu_{*}=1 / \sqrt{3}, T_{\text {irr }}=1250 \mathrm{~K}$ corresponding to the dayside average profile of a planet at 0.05 au from a sun-like star, and accounting for the albedo obtained in the numerical model. The internal temperature is $T_{\text {int }}=125 \mathrm{~K}$ and gravity $25 \mathrm{~ms}^{-2}$. The effective temperature of the studied slice of atmosphere ${ }^{1}$ is defined by $T_{\text {eff, } \mu_{*}}^{4}=T_{\text {int }}^{4}+\mu_{*} T_{\text {irr }}^{4}$. For the numerical solution, the relation between pressure and optical depth was calculated using Rosseland mean opacities; TiO and VO opacities were not included.

and the ratio of the visible to infrared opacity $\gamma_{\mathrm{v}} \equiv \kappa_{\mathrm{v}} / \kappa_{\mathrm{th}}$. For hot Jupiters, as in the example in Fig. 1, the real temperature profiles at low optical depths can be several hundreds of Kelvins cooler than predicted by the semi-grey solutions.

The levels probed both by transit spectroscopy and by the observations of secondary eclipses of exoplanets often correspond to low-optical depth levels (e.g. Burrows et al. 2007; Fortney et al. 2008; Showman et al. 2009), i.e. where semigrey models seem to systematically overestimate the temperatures. Furthermore, because the problem persists regardless of the main parameters, this implies that the functional form of the semi-grey solutions is probably not appropriate for inversion models. Non-grey effects are known to facilitate the cooling of the upper atmosphere (see Pierrehumbert 2010, for a qualitative explanation) so they must be included. This is the purpose of the present paper.

We first describe previous analytical methods used to solve the radiative transfer problem analytically. In Sect. 3, we then derive an analytical non-grey line model and compare it to previous models in Sect. 4. In Sect. 5 we study the role of non-grey effects in shaping the atmospheric thermal structure. Eventually we apply our model to the structure of irradiated giant planets in Sect. 6. We note here that while we focus the discussion on exoplanets, we believe that this model is applicable to a much wider variety of problems, as long as an atmosphere is irradiated both from above and below. Our method can also be used to solve the radiative transfer equations in other geometries, such as the thermal structure of protoplanetary disk. We provide our conclusions in Sect. 7.

\section{Assumptions and previous analytical models}

\subsection{Setting}

\subsubsection{The equation of radiative transfer}

Following Guillot (2010), we will consider the problem of a plane-parallel atmosphere in local thermodynamic equilibrium that receives from above a collimated flux $\sigma T_{\text {irr }}^{4}$ at an angle $\theta_{*}=\cos ^{-1}\left(\mu_{*}\right)$ from the vertical, and from below an isotropic flux $\sigma T_{\text {int }}^{4}$. The total energy budget of the modelled atmosphere is then set by $T_{\text {eff, } \mu_{*}}^{4}=T_{\text {int }}^{4}+\mu_{*} T_{\text {irr }}^{4}$, which defines the effective temperature in this paper ${ }^{1}$. The irradiation and intrinsic fluxes are generally characterized by very different wavelengths. Although this is not required in the solution that we propose, it is convenient to think of them as being emitted preferentially in the visible and in the infrared, respectively. Scattering processes can influence both the thermal and visible radiation. As shown by Heng et al. (2012), the solution including symmetrical scattering for the incoming radiation with the Eddington approximation is equivalent to the one obtained without scattering when the irradiation flux is reduced by a factor of $(1-A)$ and the visible opacity is reduced by a factor $1 / \sqrt{\xi}$, where $\mathrm{A}$ is the Bond albedo of the planet and $\xi$ is the ratio of the absorption to the extinction opacity (see also Meador \& Weaver 1980, for a review of the different two stream methods including scattering). In this paper, the irradiation flux and the visible opacity are treated as parameters, thus we implicitly take into account symmetrical scattering of the incoming radiation (e.g. Rayleigh scattering). Scattering of the thermal radiation, however, is neglected.

In order to solve the radiative transfer problem for a plane parallel atmosphere in local thermodynamic equilibrium, one has to solve the following equation for all frequencies $v$ and all directions $\mu$ (Chandrasekhar 1960),

$-\mu \frac{\mathrm{d} I_{\mu \nu}}{\mathrm{d} m}=\kappa_{v} I_{v \mu}-\kappa_{v} B_{v}(T)$

where $I_{\mu \nu}$ is the specific intensity at the wavelength $v$ propagating with an angle $\theta=\cos ^{-1}(\mu)$ with the vertical, $\kappa_{v}$ is the opacity at a given wavelength, $B_{v}$ is the Planck function, and $\mathrm{d} m=\rho \mathrm{d} z$ is the mass increment along the path of the radiation. As usual, $T$, $\rho$, and $z$ are the atmospheric temperature, density and height, respectively. The main difficulty in solving Eq. (1) lies in its triple dependence on $\mu, v$, and $T$ and its additional dependence on $m$. An analytical solution requires simplifications of the opacities and of the dependence of the radiation intensity on angle.

\subsubsection{Opacities and optical depth}

The need for simplification implies that mean opacities must be used. The most common one is the Rosseland mean, defined as

$$
\frac{1}{\kappa_{\mathrm{R}}} \equiv\left(\int_{0}^{\infty} \frac{\partial B_{v}}{\partial T} \mathrm{~d} v\right)^{-1} \int_{0}^{\infty} \frac{1}{\kappa_{v}} \frac{\partial B_{v}}{\partial T} \mathrm{~d} v .
$$

1 In stellar physics the effective temperature is usually what we call the internal temperature. In both planetary and stellar fields, the effective temperature aims at representing the total energy budget of the atmosphere. Although in stellar physics most of the flux comes from the deep interior, this is not true in irradiated atmospheres, and $T_{\mathrm{eff}, \mu_{*}}^{4}=T_{\mathrm{int}}^{4}+\mu_{*} T_{\mathrm{irr}}^{4}$ is a better representation of the total energy budget of the studied slice of atmosphere. The energy budget of the whole atmosphere is therefore $T_{\mathrm{eff}}^{4}=T_{\mathrm{int}}^{4}+T_{\mathrm{irr}}^{4} / 4$. 
When at all wavelengths the mean free path of photons is small compared to the scale height of the atmosphere, the radiative gradient obeys its well-defined diffusion limit and (unless convection sets in) the temperature gradient become that obtained from a grey atmosphere in which the opacity is set to the Rosseland mean (Mihalas \& Mihalas 1984, p. 350). We hence define the optical depth $\tau$ on the basis of the Rosseland mean opacity, such that, along the vertical direction

$\mathrm{d} \tau \equiv \kappa_{\mathrm{R}} \mathrm{d} m$.

Assuming hydrostatic equilibrium, the relation between pressure and optical depth can be found by integrating Eq. (3):

$\tau(P)=\int_{0}^{P} \frac{\kappa_{\mathrm{R}}\left(P^{\prime}, T\left(P^{\prime}\right)\right)}{g} \mathrm{~d} P^{\prime}$.

The optical depth thus becomes the natural variable to account for the dependence on depth in the radiative transfer problem. For any strictly positive Rosseland mean opacities, Eq. (4) is a bijection relating pressure and optical depth. Thus, solution of the radiative transfer equations in terms of optical depth can be converted to a solution in term of pressure for any functional form of the Rosseland mean opacities.

The second mean opacity that is traditionally used for radiative transfer is the so-called Planck mean:

$\kappa_{\mathrm{P}} \equiv\left(\int_{0}^{\infty} B_{v} \mathrm{~d} v\right)^{-1} \int_{0}^{\infty} \kappa_{v} B_{v} \mathrm{~d} v$

We use the ratio of the Planck and Rosseland mean opacities to quantify the non-greyness of the atmosphere:

$\gamma_{\mathrm{P}} \equiv \frac{\kappa_{\mathrm{P}}}{\kappa_{\mathrm{R}}}$.

While the value of the Rosseland mean opacity is dominated by the lowest values of the opacity function $\kappa_{v}$, the Planck mean opacity is dominated by its highest values. Thus, it can be shown that $\gamma_{\mathrm{P}}=1$ for a grey atmosphere and $\gamma_{\mathrm{P}}>1$ for a non-grey atmosphere (King 1956).

In irradiated atmospheres, a collimated flux coming from the star is absorbed at different atmospheric levels. We name $\kappa_{\mathrm{v}}$ the opacity relevant to the absorption of the stellar flux. As will be shown in Sect. 3.8, the absorption of the visible flux appears linearly in the radiative transfer equations. Thus, a solution can be found using multiple visible opacity bands $\kappa_{\mathrm{v} 1}, \kappa_{\mathrm{v} 2}$, etc.

We further define the ratio of the visible opacity to the mean (Rosseland) thermal opacity:

$\gamma_{\mathrm{v}} \equiv \kappa_{\mathrm{v}} / \kappa_{\mathrm{R}}$

In order to solve the radiative transfer problem analytically, we suppose that $\gamma_{\mathrm{v}}$ is constant with optical depth. Once $\gamma_{\mathrm{v}}$ is chosen, we can solve the equations for the visible radiation independently from the final thermal structure of the atmosphere. Of course, purely grey models are such that $\gamma_{\mathrm{v}}=1$.

\subsubsection{The picket-fence model}

It is important to note at this point that two sets of opacities with different wavelength dependences may have the same Rosseland and Planck means. We must constrain the problem further, and to this intent, we now consider the simplest possible line model,

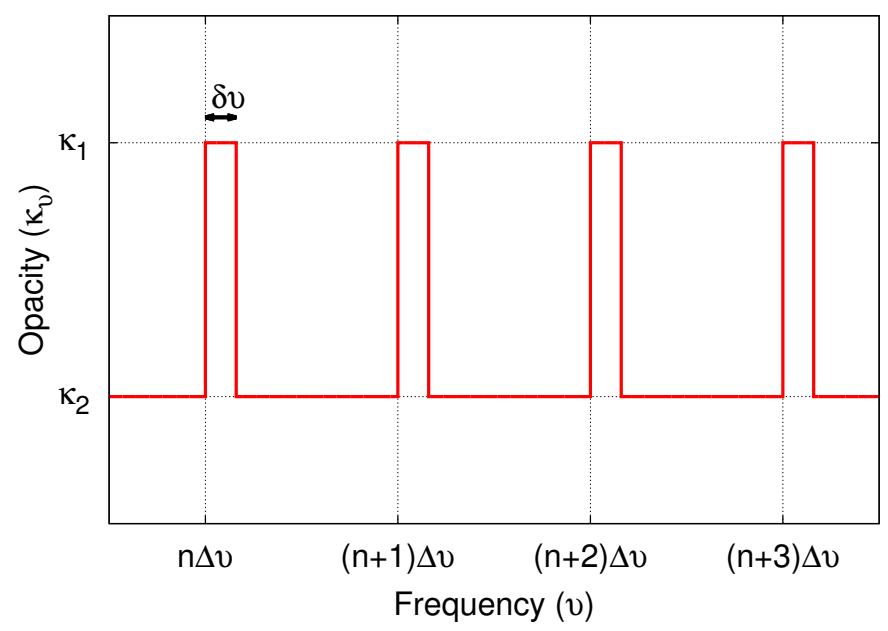

Fig. 2. Simplified thermal opacities for the picket-fence model. $\beta=$ $\delta v / \Delta v$ is the equivalent bandwidth (see text).

known as the picket-fence model (Mihalas 1978), where the thermal opacities can take two different values $\kappa_{1}$ and $\kappa_{2}$ (see Fig. 2) such that

$\kappa_{v}=\left\{\begin{array}{ll}\kappa_{1} & \text { for } v \in[n \Delta v, n \Delta v+\delta v] \\ \kappa_{2} & \text { for } v \in[n \Delta v+\delta v,(n+1) \Delta v]\end{array} \quad n \in[1, N]\right.$.

We define an equivalent bandwidth by:

$\beta=\frac{1}{\int_{0}^{\infty} B_{v} \mathrm{~d} v} \sum_{n=1}^{N} \int_{n \Delta v}^{n \Delta v+\delta v} B_{v} \mathrm{~d} v$.

The characteristic width of the Planck function can be defined as $\Delta v_{P} \equiv\left(\frac{1}{B} \frac{\partial B_{v}}{\partial v}\right)^{-1}$. When choosing $\Delta v \ll \Delta v_{P}$, the Planck function can be considered constant over $\Delta v$ and we get $\beta=\delta v / \Delta v$. The Planck and Rosseland mean opacities then become (see Eqs. (2) and (5))

$\kappa_{\mathrm{R}}=\frac{\kappa_{1} \kappa_{2}}{\beta \kappa_{2}+(1-\beta) \kappa_{1}}$

$\kappa_{\mathrm{P}}=\beta \kappa_{1}+(1-\beta) \kappa_{2}$.

We also define the following ratios:

$\gamma_{1} \equiv \kappa_{1} / \kappa_{\mathrm{R}}$

$\gamma_{2} \equiv \kappa_{2} / \kappa_{\mathrm{R}}$

$R \equiv \kappa_{1} / \kappa_{2}=\gamma_{1} / \gamma_{2}$.

Following Chandrasekhar (1935), we also define a limit optical depth

$\tau_{\lim } \equiv \frac{1}{\gamma_{1} \gamma_{2}} \sqrt{\frac{\gamma_{\mathrm{P}}}{3}}$

The role of $\tau_{\text {lim }}$ in shaping the final temperature profile is discussed in Sect. 5 and its variations with $R$ and $\beta$ is pictured in Fig. 8 .

\subsection{The method of discrete ordinates for the non-irradiated problem}

\subsubsection{The grey case}

An approximate method to solve Eq. (1) including the angular dependency has been developed by Chandrasekhar (1960) in the 
case of a non-irradiated atmosphere $\left(T_{\mathrm{irr}}=0\right)$. The idea is to replace the integrals over angle in Eq. (1) by a Gaussian sum over $\mu$. It can then be solved to an arbitrary precision by increasing the number of terms in the sum. The boundary condition at the top of the atmosphere is simply given as $I_{\mu<0}(0)=0$. The expansion to the fourth term yields the temperature profile

$T(\tau)^{4}=\frac{3 T_{\text {int }}^{4}}{4}\left(\tau+Q+L_{1} \mathrm{e}^{-k_{1} \tau}+L_{2} \mathrm{e}^{-k_{2} \tau}+L_{3} \mathrm{e}^{-k_{3} \tau}\right)$,

with $Q=0.706920, L_{1}=-0.083921, L_{2}=-0.036187, L_{3}=$ $-0.009461, k_{1}=1.103188, k_{2}=1.591778$, and $k_{3}=4.45808$ (Chandrasekhar 1960, Table VIII) $^{2}$. One of the important results from this formalism is that the skin temperature of the planet (defined as the temperature at zero optical depth) is independent of the order of expansion and therefore corresponds to the exact value

$T_{\text {skin }}^{4}=\sqrt{3} \frac{T_{\text {int }}^{4}}{4}$

This expression is exact only in the limit of a grey, non-irradiated atmosphere.

\subsubsection{The non-grey case}

Chandrasekhar (1960) also developed a perturbation method in order to include non-grey thermal opacities. This method was improved by Krook (1963). However, these perturbation methods either work for small departures from the grey opacities or involve a fastidious iterative procedure (e.g. Unno \& Yamashita 1960; Avrett \& Krook 1963) and are no longer fully analytical. However, considering that the variations in the opacities are small compared to the variations of the Planck function, analytical solutions can be found for an arbitrarily large departure from the grey opacities. Noting the similar role of $\mu$ and $\kappa_{v}$ in Eq. (1), King (1956), following Münch (1946), used the method of discrete ordinates in order to turn the integrals over frequency into Gaussian sums. For the picket-fence model defined in Sect. 2.1.3 and the second approximation for the angular dependency, King's method leads to the following temperature profile

$T^{4}(\tau)=\frac{3}{4} T_{\text {int }}^{4}\left[\frac{1}{\sqrt{3 \gamma_{\mathrm{P}}}}+\tau+\frac{\left(\sqrt{\gamma_{\mathrm{P}}}-\gamma_{1}\right)\left(\sqrt{\gamma_{\mathrm{P}}}-\gamma_{2}\right)}{\gamma_{1} \gamma_{2} \sqrt{3 \gamma_{\mathrm{P}}}}\left(\mathrm{e}^{-\tau / \tau_{\text {lim }}}-1\right)\right]$

As in the grey case, the method of discrete ordinates leads to an exact relation for the skin temperature, whatever the dependency of $\kappa_{v}$ on frequency (but no dependence on pressure or temperature):

$T_{\text {skin }}^{4}=\sqrt{\frac{3}{\gamma_{\mathrm{P}}}} \frac{T_{\mathrm{int}}^{4}}{4}$

In the grey limit, $\gamma_{\mathrm{P}}=1$ and we recover Eq. (17). Otherwise, $\gamma_{\mathrm{P}}>1$, implying that for a non-irradiated atmosphere, non-grey effects always tend to lower the atmospheric skin temperature.

\footnotetext{
2 We noticed that the values of $L_{1}$ and $L_{3}$ in Chandrasekhar's book were inverted and corrected this here.
}

\subsection{Moment equation method}

\subsubsection{Equations for the momentum of the radiation intensity}

A simpler way to solve the radiative transfer equation has been carried out by Eddington (1916). The idea is to solve the equation using the different momentum of the intensity defined as

$\left(J_{v}, H_{v}, K_{v}\right)=\frac{1}{2} \int_{-1}^{1} I_{\mu \nu}\left(1, \mu, \mu^{2}\right) \mathrm{d} \mu$.

Then, integrating over $\mu$ Eq. (1) and $\mu$ times Eq. (1) one gets the momentum equations

$\frac{\mathrm{d} H_{v}}{\mathrm{~d} \tau_{v}}=J_{v}-B_{v}(T)$.

$\frac{\mathrm{d} K_{v}}{\mathrm{~d} \tau_{v}}=H_{v}$.

Assuming the atmosphere to be in radiative equilibrium, we can write

$\int_{0}^{\infty}\left(\kappa_{v} J_{v}-\kappa_{v} B_{v}\right) \mathrm{d} v=0$.

For a grey atmosphere $\left(\kappa_{v}=\kappa_{\mathrm{R}} \forall v\right)$, Eqs. (21) $-(23)$ can be integrated over frequency, leading to an equation for $J, H, K$, and $B$, the frequency-integrated versions of $J_{v}, H_{v}, K_{v}$, and $B_{v}$. The radiative equilibrium equation becomes

$J=B$.

The frequency integrated versions of Eqs. (21)-(23) are a set of three equations with four unknowns. The system is not closed because by integrating Eq. (1) over all angles we have lost the information on the angular dependency of the irradiation. A closure relationship that contains this angular dependency is therefore needed. A common closure relationship, known as the Eddington approximation is

$J=3 K$.

This relationship is exact in two very different cases: when the radiation field is isotropic $\left(I_{\mu}\right.$ independent of $\mu$ ), and in the twostream approximation $\left(I_{\mu>0}=I_{0}^{+}\right.$and $\left.I_{\mu<0}=I_{0}^{-}\right)$. Although this seems to be a very restrictive approximation, it is relevant for the deep layers of the atmosphere because of the quasi-isotropy of the radiation field there. It is also good for the top of the atmosphere, where the flux comes mainly from the $\tau \approx 1$ layer. Indeed, the exact solution gives a ratio $J / K$ that differs by no more than $20 \%$ from the $1 / 3$ ratio over the whole atmosphere and leads to a temperature profile that is correct to $4 \%$ in the grey case (see the plain blue line in Fig. 4).

\subsubsection{Top boundary condition}

Although in the method of discrete ordinates the boundary condition at the top of the atmosphere is intuitive, in the momentum equations method it is less obvious and different choices have been made by different authors. Usually, the expression for $J$ is known and some integration constants need to be found. Two equations are needed, one for $J(0)$ and one for $H(0)$. Four possibilities are widely used in the literature from which one has to choose two:

1. The radiative equilibrium equation that relates the emergent flux at the top of the atmosphere to the internal flux from the planet and the incident flux from the star. 
2. An ad-hoc relation between $H(0)$ and $J(0)$ at $\tau=0: H(0)=$ $f_{\mathrm{H}} J(0)$, where $f_{\mathrm{H}}$ is often called the second Eddington coefficient.

3. A calculation of $H(0)$ from the second moment equation (Eq. (22)) and the Eddington approximation.

4. A calculation of $H(0)$ from the integration of the source function through the entire atmosphere, known as the Milne equation (Mihalas \& Mihalas 1984, p. 347): $H(0)=$ $\frac{1}{2} \int_{0}^{\infty} B(\tau) E_{2}(\tau) \mathrm{d} \tau$

For grey and semi-grey models, the first condition is natural and so it was used by Hansen (2008) and Guillot (2010). For the other part of the top boundary condition, Guillot (2010) chose to use the second and Hansen (2008) the fourth condition (see Appendix A of Guillot 2010 for a comparison of the two expressions).

In the case of a non-grey model, the first condition cannot be implemented (at least directly) because it is a constraint on the total thermal flux, but it provides no information on how the thermal flux is split between the opacity bands that are considered. Chandrasekhar (1935) therefore uses conditions 2 and 3 in each of the opacity bands for his non-grey, non-irradiated model. He also notes that using condition 4 instead of condition 3 should yield better results, but it leads to more complex expressions. In this work, because an accurate treatment of the flux is needed for the non-grey irradiated model, we will use conditions 2 and 4 in each of the opacity bands. All these models are discussed in the next sections and summarized in Table 1.

\subsubsection{Non-irradiated, grey case}

In this section we consider the case $T_{\text {irr }}=0$. Under the grey approximation, using the conditions 1 and 4, the temperature profile is given by (Mihalas \& Mihalas 1984, p. 357)

$T^{4}(\tau)=\frac{3}{4} T_{\text {int }}^{4}\left(\frac{2}{3}+\tau\right)$

which leads to the same solution as assuming condition 2 with $f_{\mathrm{H}}=1 / 2$. The skin temperature is then

$T_{\text {skin }}^{4}=2 \frac{T_{\text {int }}^{4}}{4}$

which differs from the exact solution (Eq. (17)) by a factor of $\sqrt{3} / 2$. Assuming $f_{\mathrm{H}}=1 / \sqrt{3}$ is thus tempting, as it leads to the correct skin temperature. Unfortunately, it leads to a temperature profile which is less accurate around $\tau \approx 1$.

\subsubsection{Non-irradiated non-grey picket-fence model}

Chandrasekhar (1935) provides solutions to the moment equations for the picket-fence model presented in Sect. 2.1.3. He assumes that the relation $H(0)=\frac{1}{f_{\mathrm{H}}} J(0)$ with $f_{\mathrm{H}}=1 / 2$, valid in the grey case under the Eddington approximation (see Eq. (27)), holds for the two thermal channels separately. Using this condition together with condition 3 , he obtains a temperature profile

$$
\begin{aligned}
T^{4}(\tau)= & \frac{3 T_{\text {int }}^{4}}{4}\left[\tau+\frac{\frac{2}{3}+\sqrt{\frac{1}{3 \gamma_{\mathrm{P}}}}}{1+\frac{1}{2} \sqrt{3 \gamma_{\mathrm{P}}}}\right] \\
& +\frac{3 T_{\text {int }}^{4}}{4}\left(\frac{\gamma_{\mathrm{P}}-1}{\sqrt{\gamma_{\mathrm{P}}}}\right) \frac{\frac{1}{\sqrt{3}}+\sqrt{\gamma_{\mathrm{P}}} \tau_{\lim }}{1+\frac{1}{2} \sqrt{3 \gamma_{\mathrm{P}}}}\left(1-\mathrm{e}^{-\tau / \tau_{\lim }}\right)
\end{aligned}
$$

and an equation for the skin temperature

$$
T_{\text {skin }}^{4}=2\left(\frac{2+\sqrt{\frac{3}{\gamma_{\mathrm{P}}}}}{2+\sqrt{3 \gamma_{\mathrm{P}}}}\right) \frac{T_{\text {int }}^{4}}{4},
$$

As expected, this equation reduces to Eq. (27) in the limit $\gamma_{\mathrm{P}}=1$. For high values of $\gamma_{P}$, this relation differs by a factor of $4 / 3$ from the exact one derived with the method of discrete ordinates [19]. As happens for the grey case, using $f_{\mathrm{H}}=1 / \sqrt{3}$ would lead to the exact solution for the skin temperature, but at the expense of the accuracy of the profile at deeper levels. Again, we note that, in the non-irradiated case, the temperature at the top of the atmosphere is determined by a single parameter, $\gamma_{\mathrm{P}}$, representing the non-greyness of the atmosphere. A comparison of the different expressions for the skin temperature is provided in Sect. 4.

\subsubsection{Irradiated semi-grey model}

In the case of irradiated atmospheres, the presence of an incoming collimated flux at the top of the atmosphere breaks the angular symmetry of the equations. The radiative transfer problem thus can no longer be solved analytically (at least not in a simple way) through the discrete ordinates technique. The momentum method is thus required.

To solve the problem, the radiation field is split into two parts: the incoming, collimated radiation field on one hand, the thermal radiation field on the other. The radiative equilibrium equation (Eq. (23)) links the two streams, as can be seen in Sect. 3.1 (see also Hansen 2008; Guillot 2010; Robinson \& Catling 2012). As mentioned in Sect. 2.1.2, when the incident radiation is at a much shorter wavelength than the thermal emission of the atmosphere, the two streams correspond to different characteristic wavelengths and may often be labelled as visible and infrared. This is not a requirement, however: the solutions apply if the radiation field corresponds to other wavelengths or if they overlap.

As discussed previously (Sect. 2.3.2), the boundary condition at the top of the model can be chosen in several ways. When using condition 2, Guillot (2010) lets the value of $f_{\mathrm{H}}$ be either $1 / 2$ or $1 / \sqrt{3}$. Those values are based on the non-irradiated case: $f_{\mathrm{H}}=1 / 2$ is the value that arises from the calculation of the angle dependence between $H(0)$ and $J(0)$ in the isotropic case, but $f_{\mathrm{H}}=1 / \sqrt{3}$ provides a skin temperature that agrees with the exact value. The two solutions differ by $\approx 3 \%$ at most (see Fig. 5 hereafter), and choosing one over another is not crucial. In any case, for an easier comparison, we provide here the solution of Guillot (2010) for $f_{\mathrm{H}}=1 / 2$

$$
\begin{aligned}
T^{4}= & \frac{3 T_{\text {int }}^{4}}{4}\left[\frac{2}{3}+\tau\right] \\
& +\frac{3 T_{\text {irr }}^{4}}{4} \mu_{*}\left[\frac{2}{3}+\frac{\mu_{*}}{\gamma_{\mathrm{v}}}+\left(\frac{\gamma_{\mathrm{v}}}{3 \mu_{*}}-\frac{\mu_{*}}{\gamma_{\mathrm{v}}}\right) \mathrm{e}^{-\gamma_{\mathrm{v}} \tau / \mu_{*}}\right],
\end{aligned}
$$

where $\mu_{*}$ is the cosine of the angle of the incident radiation. The skin temperature is

$T_{\text {skin }}^{4}=2 \frac{T_{\text {int }}^{4}+\mu_{*} T_{\text {irr }}^{4}}{4}+\gamma_{\mathrm{v}} T_{\text {irr }}^{4}$.

For $\gamma_{\mathrm{v}} \rightarrow 0$, the incident radiation is absorbed in the deep layers of the atmosphere and the skin temperature converges to the 


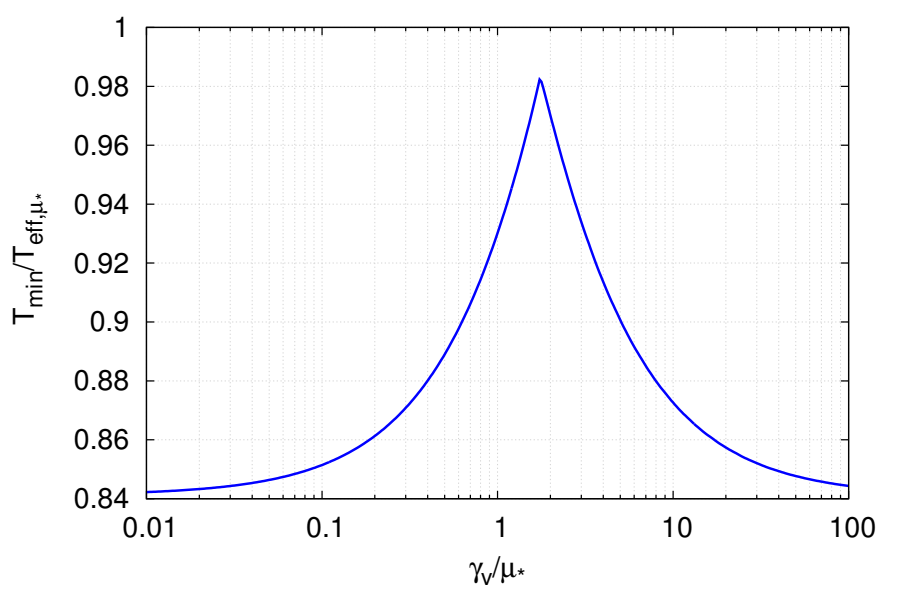

Fig. 3. Minimum temperature of the semi-grey model in terms of the effective temperature as a function of $\gamma_{\mathrm{v}} / \mu_{*}$.

skin temperature of a grey model with an effective temperature $T_{\text {eff, } \mu_{*}}^{4}=T_{\text {int }}^{4}+\mu_{*} T_{\text {irr }}^{4}$. The semi-grey model depends only on the parameter $\gamma_{\mathrm{v}}$.

As discussed in the introduction, the semi-grey model predicts minimum temperatures that are generally higher than the numerical solutions for irradiated exoplanets, independent of the choice of $\gamma_{v}$ (see Fig. 1). In fact, similarly to the skin temperature, the minimum temperature of a semi-grey atmosphere, shown in Fig. 3, depends only on the values of $T_{\text {eff, } \mu_{*}}$ and $\gamma_{\mathrm{v}}$. It is the lowest and equal to $T_{\text {eff, } \mu_{*}} / 2^{1 / 4}$ both in the $\gamma_{\mathrm{v}} \rightarrow 0$ and $\gamma_{\mathrm{v}} \rightarrow \infty$ limits. This lower bound for the semi-grey temperature profile is hotter than the one obtained by numerical calculations taking into account the full set of opacities. The discrepancy is much larger than the variations resulting from the approximation of the momentum method. Clearly, non-grey effects must be invoked to explain the low temperatures obtained by numerical models at low optical depths.

\section{An analytical irradiated non-grey picket-fence model}

\subsection{Equations}

We now derive the equations for an irradiated atmosphere in local thermodynamic equilibrium with infrared line opacities as described in Sect. 2.1.2. Thus, our model contains three different opacities: $\kappa_{1}$ and $\kappa_{2}$ for the thermal radiation and $\kappa_{\mathrm{v}}$ for the incoming radiation of the star. As explained before, the difference between the thermal and the visible channel depends on the angular dependency of the radiation and not on the frequency. Although the method of discrete ordinates is shown to lead to more exact results, it is difficult to adapt to the irradiated case. Therefore, following Chandrasekhar (1935) and Guillot (2010), we solve the radiative transfer equations using the momentum equations. Integrating Eqs. (21) and (22) over each thermal band we obtain

$$
\begin{aligned}
& \frac{\mathrm{d} H_{(1,2)}}{\mathrm{d} \tau}=\gamma_{(1,2)} J_{(1,2)}-\gamma_{(1,2)}(\beta, 1-\beta) B(T) \\
& \frac{\mathrm{d} K_{(1,2)}}{\mathrm{d} \tau}=\gamma_{(1,2)} H_{(1,2)},
\end{aligned}
$$

where the subscript indicates the integrated quantities over the given thermal band. Thus, for a quantity $X_{v}$ we have:

$$
\begin{aligned}
& X_{1}=\sum_{n} \int_{n \Delta v}^{n \Delta v+\delta v} X_{v} \mathrm{~d} v \\
& X_{2}=\sum_{n} \int_{n \Delta v+\delta_{v}}^{(n+1) \Delta v} X_{v} \mathrm{~d} v .
\end{aligned}
$$

The Planck function is considered constant over each bin of frequency $\Delta v$ and therefore $B_{1}=\beta B$ and $B_{2}=(1-\beta) B$.

We now assume that the Eddington approximation is valid in the two bands separately: $J_{(1,2)}=3 K_{(1,2)}$. Equations (32) and (33) can be combined into

$$
\begin{aligned}
& \frac{\mathrm{d}^{2} J_{1}}{\mathrm{~d} \tau^{2}}=3 \gamma_{1}^{2}\left(J_{1}-\beta B\right) \\
& \frac{\mathrm{d}^{2} J_{2}}{\mathrm{~d} \tau^{2}}=3 \gamma_{2}^{2}\left(J_{2}-(1-\beta) B\right)
\end{aligned}
$$

and the radiative equilibrium equation becomes

$\gamma_{1} J_{1}+\gamma_{2} J_{2}+\gamma_{\mathrm{v}} J_{\mathrm{v}}=\gamma_{\mathrm{P}} B$

where the quantities with subscript $\mathrm{v}$ are the momentum of the incident stellar radiation. Assuming that the incoming stellar radiation arrives as a collimated flux and hit the top of the atmosphere with an angle $\theta_{*}=\cos ^{-1} \mu_{*}$ they can are given by

$\left(J_{\mathrm{v}}, H_{\mathrm{v}}, K_{\mathrm{v}}\right)=\left(1, \mu_{*}, \mu_{*}^{2}\right) I_{*}$

with $I_{*}=\int_{0}^{\infty} I_{* v} \mathrm{~d} v$ the total incident intensity.

The absorption of the stellar irradiation can be treated separately from the thermal radiation and $J_{\mathrm{v}}$ is given by Eq. (13) of Guillot (2010),

$J_{\mathrm{v}}(\tau)=-\frac{H_{\mathrm{v}}(0)}{\mu_{*}} \mathrm{e}^{-\gamma_{\mathrm{v}}^{*} \tau}$,

where we have simplified the notation by introducing the parameter $\gamma_{\mathrm{v}}^{*} \equiv \gamma_{\mathrm{v}} / \mu_{*}$.

Equations (36) to (38) are a set of three coupled equations with three unknowns $J_{1}, J_{2}$, and $B$. In order to decouple these equations we define two new variables:

$$
\left\{\begin{array}{c}
J_{\gamma} \equiv \frac{J_{1}}{\gamma_{1}}+\frac{J_{2}}{\gamma_{2}} \\
J_{\gamma^{3}} \equiv \frac{J_{1}}{\gamma_{1}^{3}}+\frac{J_{2}}{\gamma_{2}^{3}} .
\end{array}\right.
$$

Conversely, we can come back to the original variables:

$$
\left\{\begin{array}{l}
J_{1}=-\frac{\gamma_{1}^{3}\left(\gamma_{2}^{2} J_{\gamma^{3}}-J_{\gamma}\right)}{\gamma_{1}^{2}-\gamma_{2}^{2}} \\
J_{2}=\frac{\gamma_{2}^{3}\left(\gamma_{1}^{2} J_{\gamma^{3}}-J_{\gamma}\right)}{\gamma_{1}^{2}-\gamma_{2}^{2}}
\end{array}\right.
$$

Using the combination of Eqs. $\frac{1}{\gamma_{1}}(36)+\frac{1}{\gamma_{2}}(37)$ and (38) we get $\frac{\mathrm{d}^{2} J_{\gamma}}{\mathrm{d} \tau^{2}}=-3 \gamma_{\mathrm{v}} J_{\mathrm{v}}$ 
V. Parmentier and T. Guillot: A non-grey analytical model for irradiated atmospheres. I.

Table 1. Summary of the different models compared in this paper.

\begin{tabular}{lccccc}
\hline \hline Model & $\begin{array}{c}\text { External } \\
\text { irradiation }\end{array}$ & $\begin{array}{c}\text { Eddington } \\
\text { approx. }\end{array}$ & $\begin{array}{c}\text { Non-grey } \\
\text { thermal } \\
\text { opacities }\end{array}$ & $\begin{array}{c}\text { Top boundary } \\
\text { condition }\end{array}$ & Expression \\
\hline Numerical & $\sqrt{ }$ & $\times$ & $\sqrt{ }$ & $I(0)=I_{\text {star }}$ for $\mu<0$ & N/A \\
King (1955) & $\times$ & $\times$ & $\sqrt{ }$ & $I(0)=0$ for $\mu<0$ & Eq. (18) \\
Chandrasekhar (1935) & $\times$ & $\sqrt{ }$ & $\sqrt{ }$ & Eq. (22) \& $f_{\mathrm{H}}=1 / 2$ & Eq. (28) \\
Hansen (2008) & $\sqrt{ }$ & $\sqrt{ }$ & $\times$ & Rad. Eq., \& Eq. (50) & - \\
Guillot (2010), $f_{\mathrm{H}}=1 / 2$ & $\sqrt{ }$ & $\sqrt{ }$ & $\times$ & Rad. Eq., \& $f_{\mathrm{H}}=1 / 2$ & Eq. (30) \\
Guillot (2010), $f_{\mathrm{H}}=1 / \sqrt{3}$ & $\sqrt{ }$ & $\sqrt{ }$ & $\times$ & Rad. Eq., \& $f_{\mathrm{H}}=1 / \sqrt{3}$ & - \\
This model & $\sqrt{ }$ & $\sqrt{ }$ & $\sqrt{ }$ & Eq. (50) $\& f_{\mathrm{H}}=1 / 2$ & Eq. (76) \\
\hline
\end{tabular}

The combination of Eqs. $\frac{1}{\gamma_{1}^{3}}(36)+\frac{1}{\gamma_{2}^{3}}(37)$ yields

$\frac{\mathrm{d}^{2} J_{\gamma^{3}}}{\mathrm{~d} \tau^{2}}=3\left(J_{\gamma}-B\right)$

Noting that $\gamma_{1} J_{1}+\gamma_{2} J_{2}=\left(\gamma_{1}^{2}+\gamma_{2}^{2}\right) J_{\gamma}-\left(\gamma_{1} \gamma_{2}\right)^{2} J_{\gamma^{3}}$, Eq. (38) becomes

$B=\frac{\gamma_{1}^{2}+\gamma_{2}^{2}}{\gamma_{\mathrm{P}}} J_{\gamma}-\frac{\left(\gamma_{1} \gamma_{2}\right)^{2}}{\gamma_{\mathrm{P}}} J_{\gamma^{3}}+\frac{\gamma_{\mathrm{v}}}{\gamma_{\mathrm{P}}} J_{\mathrm{v}}$

Equations (43)-(45) are now a set of two uncoupled differential equations and a linear equation.

\subsection{Boundary conditions}

To solve the differential equations we need to specify the boundary conditions. When $\tau \rightarrow+\infty$ we want to fulfill the diffusion approximation $J_{v}=B_{v}$ (Mihalas \& Mihalas 1984, p. 350). In our case this translates to $J_{1}=\beta B$ and $J_{2}=(1-\beta) B$. Furthermore, at these levels, the gradient of B should also obey the diffusion approximation (Mihalas \& Mihalas 1984)

$\frac{\mathrm{d} B}{\mathrm{~d} \tau} \underset{\tau \rightarrow+\infty}{\sim} 3 H_{\infty}$

where $4 \pi H_{\infty}=\sigma T_{\text {int }}^{4}$ is the thermal flux coming from the interior of the planet. Using the system of Eqs. (41) and noting that $\frac{\beta}{\gamma_{1}}+$ $\frac{1-\beta}{\gamma_{2}}=1$, we can derive a condition on $J_{\gamma}$ and $J_{\gamma^{3}}$ :

$\frac{\mathrm{d} J_{\gamma}}{\mathrm{d} \tau} \underset{\tau \rightarrow+\infty}{\sim} 3 H_{\infty}$

$\frac{\mathrm{d} J_{\gamma^{3}}}{\mathrm{~d} \tau} \underset{\tau \rightarrow+\infty}{\sim}\left(\frac{\beta}{\gamma_{1}^{3}}+\frac{1-\beta}{\gamma_{2}^{3}}\right) 3 H_{\infty}$.

For $\tau \rightarrow 0$ we specify the geometry of the intensity by setting:

$J_{(1,2)}(0)=2 H_{(1,2)}(0)$.

Furthermore, we calculate the flux at the top of the atmosphere in each band using Eq. (79.21) from Mihalas \& Mihalas (1984). From the assumption of local thermodynamic equilibrium, the source function in the two bands is $S_{1}\left(\tau_{1}\right)=\beta B\left(\tau / \gamma_{1}\right)$ and $S_{2}\left(\tau_{2}\right)=(1-\beta) B\left(\tau / \gamma_{2}\right)$. The upper boundary condition on the flux of the two bands thus becomes

$H_{(1,2)}(0)=\frac{1}{2} \int_{0}^{\infty}(\beta,(1-\beta)) B\left(\frac{\tau}{\gamma_{(1,2)}}\right) E_{2}(\tau) \mathrm{d} \tau$.

\subsection{Solution}

The solution of a second-order differential equation with constant coefficient is the sum of the solutions of the homogeneous equation and a particular solution of the complete equation. Thus, solutions of Eq. (43) must be of the form:

$J_{\gamma}(\tau)=C_{1}+C_{2} \tau+\frac{3}{\gamma_{\mathrm{v}}^{*}} H_{\mathrm{v}}(0) \mathrm{e}^{-\gamma_{\mathrm{v}}^{*} \tau}$.

Applying the boundary condition Eq. (47), we get $C_{2}=3 H$. For $\tau=0$ we obtain

$J_{\gamma}(0)=C_{1}+\frac{3}{\gamma_{\mathrm{v}}^{*}} H_{\mathrm{v}}(0)$.

Using Eq. (45) to eliminate $B$ and replacing $J_{\gamma}$ by its solution, Eq. (44) becomes:

$$
\begin{aligned}
\frac{\mathrm{d}^{2} J_{\gamma^{3}}}{\mathrm{~d} \tau^{2}}-3 \frac{\left(\gamma_{1} \gamma_{2}\right)^{2}}{\gamma_{\mathrm{P}}} J_{\gamma^{3}}= & 3\left(1-\frac{\gamma_{1}^{2}+\gamma_{2}^{2}}{\gamma_{\mathrm{P}}}\right)\left(C_{1}+3 H \tau\right) \\
& +3\left(1-\frac{\gamma_{1}^{2}+\gamma_{2}^{2}}{\gamma_{\mathrm{P}}}\right) \frac{3}{\gamma_{\mathrm{v}}^{*}} H_{\mathrm{v}}(0) \mathrm{e}^{-\gamma_{\mathrm{v}}^{*} \tau} \\
& +3 \frac{\gamma_{\mathrm{v}}^{*}}{\gamma_{\mathrm{P}}} H_{\mathrm{v}}(0) \mathrm{e}^{-\gamma_{\mathrm{v}}^{*} \tau}
\end{aligned}
$$

Again, solutions of this differential equation must be the sum of the solutions of the homogeneous equation and one solution of the complete equation. The homogeneous solution must have the form

$J_{\gamma^{3} \mathrm{H}}=C_{3} \mathrm{e}^{-\tau / \tau_{\lim }}+C_{4} \mathrm{e}^{+\tau / \tau_{\lim }}$,

where $C_{3}$ and $C_{4}$ are constants of integration to be determined using the boundary conditions. We look for a particular solution formed by the superposition of an exponential and an affine function. The affine function must then be a solution of Eq. (53) with $H_{\mathrm{v}}(0)=0$

$J_{\gamma^{3} \mathrm{P} 1}=-\frac{\gamma_{\mathrm{P}}}{\left(\gamma_{1} \gamma_{2}\right)^{2}}\left(1-\frac{\gamma_{1}^{2}+\gamma_{2}^{2}}{\gamma_{\mathrm{P}}}\right)\left(C_{1}+3 H \tau\right)$,

and the exponential function must be solution of Eq. (53), keeping only the exponential part on the right-hand side

$J_{\gamma^{3} \mathrm{P} 2}=\frac{\gamma_{\mathrm{P}}}{\left(\gamma_{1} \gamma_{2}\right)^{2}} \frac{1}{\left(\gamma_{\mathrm{v}}^{*} \tau_{\lim }\right)^{2}-1}\left[\left(1-\frac{\gamma_{1}^{2}+\gamma_{2}^{2}}{\gamma_{\mathrm{P}}}\right) \frac{3}{\gamma_{\mathrm{v}}^{*}}+\frac{\gamma_{\mathrm{v}}^{*}}{\gamma_{\mathrm{P}}}\right] H_{\mathrm{v}}(0) \mathrm{e}^{-\gamma_{\mathrm{v}}^{*} \tau}$. 
Applying the boundary condition defined by Eq. (48) to the full solution $J_{\gamma^{3}}=J_{\gamma^{3} \mathrm{P} 1}+J_{\gamma^{3} \mathrm{P} 2}+J_{\gamma^{3} \mathrm{H}}$, we find $C_{4}=0$. The full solution of Eq. (44) is therefore given by

$J_{\gamma^{3}}(\tau)=J_{\gamma^{3} \mathrm{P} 1}+J_{\gamma^{3} \mathrm{P} 2}+C_{3} \mathrm{e}^{-\tau / \tau_{\mathrm{lim}}}$

We can get an expression for the source function by replacing $J_{\gamma}$ and $J_{\gamma^{3}}$ in the radiative equilibrium equation (Eq. (45)):

$$
\begin{aligned}
B= & C_{1}+3 H \tau-\frac{\left(\gamma_{1} \gamma_{2}\right)^{2}}{\gamma_{\mathrm{P}}} C_{3} \mathrm{e}^{-\tau / \tau_{\lim }} \\
& +\frac{\left[3-\left(\gamma_{\mathrm{v}}^{*} / \gamma_{1}\right)^{2}\right]\left[3-\left(\gamma_{\mathrm{v}}^{*} / \gamma_{2}\right)^{2}\right]}{3 \gamma_{\mathrm{v}}^{*}\left(1-\gamma_{\mathrm{v}}^{* 2} \tau_{\lim }^{2}\right)} H_{\mathrm{v}}(0) \mathrm{e}^{-\gamma_{\mathrm{v}}^{*} \tau}
\end{aligned}
$$

To get the complete solution of the problem, we need to determine the two remaining integration constants $C_{1}$ and $C_{3}$ using the boundary condition (49). For that we need to calculate $J_{1}(0)$, $J_{2}(0), H_{1}(0)$ and $H_{2}(0)$. The first two quantities can be evaluated by inserting the values of $J_{\gamma}(0)$ and $J_{\gamma^{3}}(0)$ from Eqs. (51) and (57) into system (42):

$$
\begin{aligned}
J_{1}(0)= & -\frac{\gamma_{1}\left(\gamma_{2}-1\right)}{\gamma_{1}-\gamma_{2}} C_{1} \\
& +\frac{\gamma_{1}\left(3\left(\gamma_{1}+\gamma_{2}\right)\left(\gamma_{2}-1\right)-\gamma_{\mathrm{v}}^{* 2}+3 \gamma_{1}^{2} \gamma_{\mathrm{v}}^{* 2} \tau_{\lim }^{2}\right)}{\left(\gamma_{1}^{2}-\gamma_{2}^{2}\right) \gamma_{\mathrm{v}}^{*}\left(\gamma_{\mathrm{v}}^{* 2} \tau_{\lim }^{2}-1\right)} H_{\mathrm{v}}(0)(59) \\
& -\frac{\gamma_{1}^{3} \gamma_{2}^{2}}{\gamma_{1}^{2}-\gamma_{2}^{2}} C_{3} \\
J_{2}(0)= & \frac{\gamma_{2}\left(\gamma_{1}-1\right)}{\gamma_{1}-\gamma_{2}} C_{1} \\
& -\frac{\gamma_{2}\left(3\left(\gamma_{1}+\gamma_{2}\right)\left(\gamma_{1}-1\right)-\gamma_{\mathrm{v}}^{* 2}+3 \gamma_{2}^{2} \gamma_{\mathrm{v}}^{* 2} \tau_{\lim }^{2}\right)}{\left(\gamma_{1}^{2}-\gamma_{2}^{2}\right) \gamma_{\mathrm{v}}^{*}\left(\gamma_{\mathrm{v}}^{* 2} \tau_{\lim }^{2}-1\right)} H_{\mathrm{v}}(0)(60) \\
& +\frac{\gamma_{2}^{3} \gamma_{1}^{2}}{\gamma_{1}^{2}-\gamma_{2}^{2}} C_{3} .
\end{aligned}
$$

Noting that

$$
\begin{aligned}
& \int_{0}^{\infty} E_{2}(\tau) \mathrm{d} \tau=\frac{1}{2} \\
& \int_{0}^{\infty} \tau E_{2}(\tau) \mathrm{d} \tau=\frac{1}{3} \\
& \int_{0}^{\infty} \mathrm{e}^{-\alpha \tau} E_{2}(\tau) \mathrm{d} \tau=-\frac{1}{2}+\frac{1}{\alpha}-\frac{\ln (1+\alpha)}{\alpha^{2}},
\end{aligned}
$$

we can evaluate $H_{1}(0)$ and $H_{2}(0)$ by inserting Eqs. (58) into (50). Then, Eq. (49) is a linear system of two equations with two unknowns. After some calculations we get the expressions for $C_{1}$ and $C_{3}$

$C_{1}=\left(a_{0}+a_{1} b_{0}\right) H+\left(a_{1} b_{0} b_{1}\left(1+b_{2}+b_{3}\right)+a_{2}+a_{3}\right) H_{\mathrm{v}}(0)$

$C_{3}=b_{0} H+b_{0} b_{1}\left(1+b_{2}+b_{3}\right) H_{\mathrm{v}}(0)$, where we have

$a_{0}=\frac{1}{\gamma_{1}}+\frac{1}{\gamma_{2}}$

$a_{1}=-\frac{1}{3 \tau_{\lim }^{2}}\left[\frac{\gamma_{\mathrm{p}}}{1-\gamma_{\mathrm{p}}} \frac{\gamma_{1}+\gamma_{2}-2}{\gamma_{1}+\gamma_{2}}+\left(\gamma_{1}+\gamma_{2}\right) \tau_{\lim }-\left(A_{\mathrm{t}, 1}+A_{\mathrm{t}, 2}\right) \tau_{\lim }^{2}\right]$

$$
\begin{aligned}
a_{2}= & \frac{\tau_{\lim }^{2}}{\gamma_{\mathrm{P}} \gamma_{\mathrm{v}}^{* 2}} \\
& \times \frac{\left(3 \gamma_{1}^{2}-\gamma_{\mathrm{v}}^{* 2}\right)\left(3 \gamma_{2}^{2}-\gamma_{\mathrm{v}}^{* 2}\right)\left(\gamma_{1}+\gamma_{2}\right)-3 \gamma_{\mathrm{v}}^{*}\left(6 \gamma_{1}^{2} \gamma_{2}^{2}-\gamma_{\mathrm{v}}^{* 2}\left(\gamma_{1}^{2}+\gamma_{2}^{2}\right)\right)}{1-\gamma_{\mathrm{v}}^{* 2} \tau_{\lim }^{2}}
\end{aligned}
$$

$a_{3}=-\frac{\tau_{\lim }^{2}\left(3 \gamma_{1}^{2}-\gamma_{\mathrm{v}}^{* 2}\right)\left(3 \gamma_{2}^{2}-\gamma_{\mathrm{v}}^{* 2}\right)\left(A_{\mathrm{v}, 2}+A_{\mathrm{v}, 1}\right)}{\gamma_{\mathrm{P}} \gamma_{\mathrm{v}}^{* 3}\left(1-\gamma_{\mathrm{v}}^{* 2} \tau_{\lim }^{2}\right)}$

$b_{0}=\left(\frac{\gamma_{1} \gamma_{2}}{\gamma_{1}-\gamma_{2}} \frac{A_{\mathrm{t}, 1}-A_{\mathrm{t}, 2}}{3}-\frac{\left(\gamma_{1} \gamma_{2}\right)^{2}}{\sqrt{3 \gamma_{\mathrm{P}}}}-\frac{\left(\gamma_{1} \gamma_{2}\right)^{3}}{\left(1-\gamma_{1}\right)\left(1-\gamma_{2}\right)\left(\gamma_{1}+\gamma_{2}\right)}\right)^{-1}$

$b_{1}=\frac{\gamma_{1} \gamma_{2}\left(3 \gamma_{1}^{2}-\gamma_{\mathrm{v}}^{* 2}\right)\left(3 \gamma_{2}^{2}-\gamma_{\mathrm{v}}^{* 2}\right) \tau_{\lim }^{2}}{\gamma_{\mathrm{P}} \gamma_{\mathrm{v}}^{* 2}\left(\gamma_{\mathrm{v}}^{* 2} \tau_{\lim }^{2}-1\right)}$

$b_{2}=\frac{3\left(\gamma_{1}+\gamma_{2}\right) \gamma_{\mathrm{v}}^{* 3}}{\left(3 \gamma_{1}^{2}-\gamma_{\mathrm{v}}^{* 2}\right)\left(3 \gamma_{2}^{2}-\gamma_{\mathrm{v}}^{* 2}\right)}$

$b_{3}=\frac{A_{\mathrm{v}, 2}-A_{\mathrm{v}, 1}}{\gamma_{\mathrm{v}}^{*}\left(\gamma_{1}-\gamma_{2}\right)}$,

where we defined

$A_{\mathrm{t}, i}=\gamma_{i}^{2} \ln \left(1+\frac{1}{\tau_{\lim } \gamma_{i}}\right)$

$A_{\mathrm{v}, i}=\gamma_{i}^{2} \ln \left(1+\frac{\gamma_{\mathrm{v}}^{*}}{\gamma_{i}}\right)$.

\subsection{Atmospheric temperature profile}

Using the relations $B=\sigma T^{4} / \pi, H=\sigma T_{\text {int }}^{4} / 4 \pi$, and $H_{\mathrm{v}}(0)=$ $-\mu_{*} \sigma T_{\text {irr }}^{4} / 4 \pi$ and Eq. (58), we can derive the equation for the temperature at any optical depth

$$
\begin{aligned}
T^{4}= & \frac{3 T_{\text {int }}^{4}}{4}\left(\tau+A+B \mathrm{e}^{-\tau / \tau_{\lim }}\right) \\
& +\frac{3 T_{\text {irr }}^{4}}{4} \mu_{*}\left(C+D \mathrm{e}^{-\tau / \tau_{\lim }}+E \mathrm{e}^{-\gamma_{v}^{*} \tau}\right)
\end{aligned}
$$

with

$A=\frac{1}{3}\left(a_{0}+a_{1} b_{0}\right)$

$B=-\frac{1}{3} \frac{\left(\gamma_{1} \gamma_{2}\right)^{2}}{\gamma_{\mathrm{p}}} b_{0}$

$C=-\frac{1}{3}\left[b_{0} b_{1}\left(1+b_{2}+b_{3}\right) a_{1}+a_{2}+a_{3}\right]$

$D=\frac{1}{3} \frac{\left(\gamma_{1} \gamma_{2}\right)^{2}}{\gamma_{\mathrm{p}}} b_{0} b_{1}\left(1+b_{2}+b_{3}\right)$

$E=\frac{\left[3-\left(\gamma_{\mathrm{v}}^{*} / \gamma_{1}\right)^{2}\right]\left[3-\left(\gamma_{\mathrm{v}}^{*} / \gamma_{2}\right)^{2}\right]}{9 \gamma_{\mathrm{v}}^{*}\left[\left(\gamma_{\mathrm{v}}^{*} \tau_{\lim }\right)^{2}-1\right]}$ 
V. Parmentier and T. Guillot: A non-grey analytical model for irradiated atmospheres. I.

\subsection{Grey limit}

In the grey limit, $\gamma_{\mathrm{P}} \rightarrow 1$ (as $\gamma_{1}$ and $\gamma_{2}$ ) and we obtain

$$
\begin{aligned}
& A \rightarrow 2 / 3 \\
& B \rightarrow 0 \\
& C \rightarrow 2 / 3-2 / \gamma_{\mathrm{v}}^{* 2}+2 / \gamma_{\mathrm{v}}^{*}+2 \log \left(1+\gamma_{\mathrm{v}}^{*}\right)\left(1 / \gamma_{\mathrm{v}}^{* 3}-1 /\left(3 \gamma_{\mathrm{v}}^{*}\right)\right) \\
& D \rightarrow 0 \\
& E \rightarrow \gamma_{\mathrm{v}}^{*} / 3-1 / \gamma_{\mathrm{v}}^{*} .
\end{aligned}
$$

If we also assume that $\gamma_{\mathrm{v}}^{*} \rightarrow 0$ we obtain $C \rightarrow 2 / 3+1 / \gamma_{\mathrm{v}}^{*}$ and $E \rightarrow-1 / \gamma_{\mathrm{v}}^{*}$ and the solution converges towards that of Guillot (2010) (see Eq. (30)). For other values of $\gamma_{\mathrm{v}}^{*}$ our model differs from the solutions of Guillot (2010, see also Hansen 2008) because of the different boundary conditions used in the two models (see Sect. 2.3.2). However, calculations show that the value of $C$ obtained here differs from the same coefficient extracted from Eq. (30) by at most $12 \%$ and that the two solutions also converge for $\gamma_{\mathrm{v}}^{*} \rightarrow \infty$. As seen in Fig. 5, in the semi-grey limit, and when calculating the full temperature profile, our model differs by at most $2 \%$ from the Guillot (2010) model. The difference between the various solutions must be attributed to the Eddington approximation.

\subsection{Using the model}

The temperature vs. optical depth profile for our irradiated picket-fence model is given by Eq. (76). The profile has been derived using the Rosseland optical depth as vertical coordinate. It is therefore valid for any functional form of the Rosseland opacities. Equation (4) allows us to switch from $\tau$ to $P$ as the vertical coordinate. Although, for convenience, this expression contains four different variables, $\gamma_{1}, \gamma_{2}, \gamma_{\mathrm{P}}$, and $\tau_{\text {lim }}$, it must be kept in mind that, besides the Rosseland mean opacity, there are only two independent variables in the problem. The variables $\beta$ and $R \equiv \gamma_{1} / \gamma_{2}=\kappa_{1} / \kappa_{2}$ are the ones to consider to control the shape of the thermal opacities. The variables $\gamma_{\mathrm{P}}$ and $\tau_{\lim }$ are the ones to consider to control the profile itself. The variable $\gamma_{\mathrm{P}}$ is directly related to the skin temperature of the planet (see Sect. 4.3) whereas $\tau_{\lim }$ is the optical depth at which the irradiated picketfence model differs from the semi-grey model. The steps to use our model are as follow:

1) choose the pair of variables suitable for the problem: $(R, \beta)$ or $\left(\gamma_{\mathrm{P}}, \tau_{\text {lim }}\right)$, for example;

2) using Eqs. (87) to (95), calculate the values of $\gamma_{\mathrm{P}}, \gamma_{1}, \gamma_{2}$, and $\tau_{\text {lim }}$

3) using Eqs (77) to (81) and Eqs. (66) to (75), calculate the coefficients A, B, C, D, and E.;

4) using Eq. (76), calculate the temperature/optical depth profile;

5) using Eq. (4), calculate the pressure/optical depth relationship and therefore the pressure/temperature profile.

For Rosseland opacities depending on the temperature, step 5) can be iterated until convergence. Given the apparent complexity of the solution, we provide a ready-to-use code ${ }^{3}$ in different languages that gives the temperature/optical depth profile (steps 1 to 4 ) or the temperature/pressure profile given a Rosseland mean opacity.

\footnotetext{
https://www.oca.eu/parmentier/nongrey
}

The relationship between the different variables are listed below:

$$
\begin{aligned}
& \gamma_{\mathrm{P}}=\beta+R-\beta R+\frac{\beta+R-\beta R}{R}-\frac{(\beta+R-\beta R)^{2}}{R} \\
& \tau_{\lim }=\frac{\sqrt{R} \sqrt{\beta(R-1)^{2}-\beta^{2}(R-1)^{2}+R}}{\sqrt{3}(\beta+R-\beta R)^{2}} \\
& R=\frac{\sqrt{3 \gamma_{\mathrm{P}}}+3 \gamma_{\mathrm{P}} \tau_{\lim }+\sqrt{\Delta}}{\sqrt{3 \gamma_{\mathrm{P}}}+3 \gamma_{\mathrm{P}} \tau_{\lim }-\sqrt{\Delta}} \\
& \beta=\frac{\sqrt{\Delta}-\sqrt{3 \gamma_{\mathrm{P}}}+3 \gamma_{\mathrm{P}} \tau_{\lim }}{2 \sqrt{\Delta}} \\
& \Delta=3 \gamma_{\mathrm{P}}+3 \sqrt{\gamma_{\mathrm{P}}} \tau_{\lim }\left(2 \sqrt{3} \gamma_{\mathrm{P}}+3 \gamma_{\mathrm{P}}^{3 / 2} \tau_{\lim }-4 \sqrt{3}\right) \\
& \gamma_{1}=\beta+R-\beta R \\
& \gamma_{2}=\frac{\beta+R-\beta R}{R} \\
& \gamma_{1}=\frac{\sqrt{3 \gamma_{\mathrm{P}}}+3 \gamma_{\mathrm{P}} \tau_{\lim }+\sqrt{\Delta}}{6 \tau_{\lim }} \\
& \gamma_{2}=\frac{\sqrt{3 \gamma_{\mathrm{P}}}+3 \gamma_{\mathrm{P}} \tau_{\lim }-\sqrt{\Delta}}{6 \tau_{\lim }} \\
& R=1+\frac{\gamma_{\mathrm{P}}-1}{2 \beta(1-\beta)}+\sqrt{\left(\frac{\gamma_{\mathrm{P}}-1}{2 \beta(1-\beta)}\right)^{2}+\frac{\gamma_{\mathrm{P}}-1}{2 \beta(1-\beta)}} .
\end{aligned}
$$

\subsection{About averaging}

Equation (76) can thus be considered to depend on $\kappa_{\mathrm{R}}, \gamma_{\mathrm{P}} \equiv$ $\kappa_{\mathrm{P}} / \kappa_{\mathrm{R}}$, and $\beta$. While $\kappa_{\mathrm{R}}$ can be considered a function of pressure and temperature (e.g. extracted from a known Rosseland opacity table) when deriving the atmospheric temperature profile, it is important to realize that the analytical solution remains valid only if $\gamma_{\mathrm{P}}$ and $\beta$ are held constant. This analytical solution therefore cannot accommodate consistent Rosseland and Planck opacities as a function of depth. A solution consisting of atmospheric slices with different values of $\gamma_{\mathrm{P}}$ have been derived by Chandrasekhar (1935) for the non-irradiated case, but it becomes too complex to be handled easily.

Furthermore, the solution is provided only for one fixed direction of the incoming irradiation. When considering the case of a non-resolved planet around a star, any information acquired on its atmosphere will have been averaged over at least a fraction of its surface. Solving this problem for the particular case of Eq. (76) goes beyond the scope of the present work, but it can be approximated relatively well on the basis of the study by Guillot (2010). This work shows that given an irradiation flux at the substellar point $\sigma T_{\text {sub }}^{4} \equiv \sigma\left(R_{*} / D\right)^{2} T_{*}^{4}$, where $T_{*}$ is the star's effective temperature, $R_{*}$ its radius, and $D$ the star-planet distance, the average temperature profile of the planet will be very close to that obtained from the one-dimensional solution with an average angle $\mu^{*}=1 / \sqrt{3}$ and an average irradiation temperature $T_{\text {irr }}=(1-A)^{1 / 4} f^{1 / 4} T_{\text {sub }}$, where $A$ is the (assumed) Bond albedo of the atmosphere and $f$ is a correction factor, equal to $1 / 4$ when averaging on the entire surface of the planet and equal to $1 / 2$ when averaging on the dayside only. This corresponds to the socalled isotropic approximation. In the semi-grey case, it is found to be within $2 \%$ of the actual average for a typical hot-Jupiter (see Fig. 2 of Guillot 2010). 
For the interpretation of spectroscopic and photometric data of secondary eclipses, the dayside average is often used $(f=$ $1 / 2$ ). For the calculation of evolution models, the global average is the correct physical quantity to be used when the composition and opacity variations in latitude and longitude are not precisely known (see Guillot 2010). In that case, $f=1 / 4$ which is equivalent to setting the irradiation temperature equal to the usual equilibrium temperature defined as $T_{\text {eq }} \equiv T_{*}\left(R_{*} / 2 D\right)^{1 / 2}$ (Saumon et al. 1996). Obviously detailed interpretations must use an approach mixing three-dimensional dynamical and radiative transfer models (see Guillot 2010; Heng et al. 2012; Showman et al. 2009).

\subsection{Adding several bands in the visible}

Although, for the simplicity of the derivation, our model used only one spectral band in the visible channel, it can be easily extended to $n$ visible bands. The most important point is that our equations, and in particular Eq. (43), are linear in the visible. Thus, the equations can be solved for any linear combination of visible bands. In that case the first momentum of the visible intensity (see Eq. (40)) writes

$J_{\mathrm{v}}(\tau)=-\frac{H_{\mathrm{v}}(0)}{\mu_{*}} \sum_{i=1}^{n} \beta_{\mathrm{v} i} \mathrm{e}^{-\gamma_{\mathrm{v} i}^{*} \tau}$,

where $\beta_{\mathrm{v} i}$ is the relative spectral extent of the $i$ th band and $\gamma_{\mathrm{v} i}=$ $\kappa_{\mathrm{v} i} / \kappa_{\mathrm{R}}$ with $\kappa_{\mathrm{v} i}$ the opacity in the $i$ th visible band. Equation (76) then becomes

$$
\begin{aligned}
T^{4}= & \frac{3 T_{\text {int }}^{4}}{4}\left(\tau+A+B \mathrm{e}^{-\tau / \tau_{\text {lim }}}\right) \\
& +\sum_{i=1}^{n} \frac{3 \beta_{\mathrm{v} i} T_{\text {irr }}^{4}}{4} \mu_{*}\left(C_{i}+D_{i} \mathrm{e}^{-\tau / \tau_{\lim }}+E_{i} \mathrm{e}^{-\gamma_{\mathrm{vi}}^{*} \tau}\right)
\end{aligned}
$$

where $C_{i}, D_{i}$, and $E_{i}$ are the coefficients $C, D$, and $E$ given by Eqs. (79) to (81) where $\gamma_{\mathrm{v}}^{*}$ have been replaced by $\gamma_{\mathrm{v} i}^{*}$.

\section{Comparisons}

\subsection{Comparison of non-irradiated solutions}

Figure 4 shows a comparison between our results and the solutions of King (1955) and Chandrasekhar (1935). The solutions are extremely close, the temperatures being always less than a few percentage points of each other. Our solution is almost identical to that of Chandrasekhar (1935), a consequence of using the Eddington approximation and similar boundary conditions. The difference of these with the exact solution from King (1955) can be attributed to the Eddington approximation.

The non-grey effects lead to colder temperatures at small optical depths. When $\beta$ is close to unity, a blanketing effect leads to a heating of the deeper layers too. All solutions have the correct behaviour (see also Sect. 5).

\subsection{Comparison of irradiated solutions}

The solutions presented in this work for the irradiated semi-grey case (i.e. $R \equiv \kappa_{1} / \kappa_{2}=1$ ) are very similar to those of Guillot (2010). As seen in Fig. 5, the solutions obtained either with $f_{\mathrm{H}}=1 / 2$, or $f_{\mathrm{H}}=1 / \sqrt{3}$ have relative differences of up to $2 \%$ with those of this work. These differences are of the same kind as those arising from the use of the Eddington approximation compared to exact solutions discussed previously. They are inherent

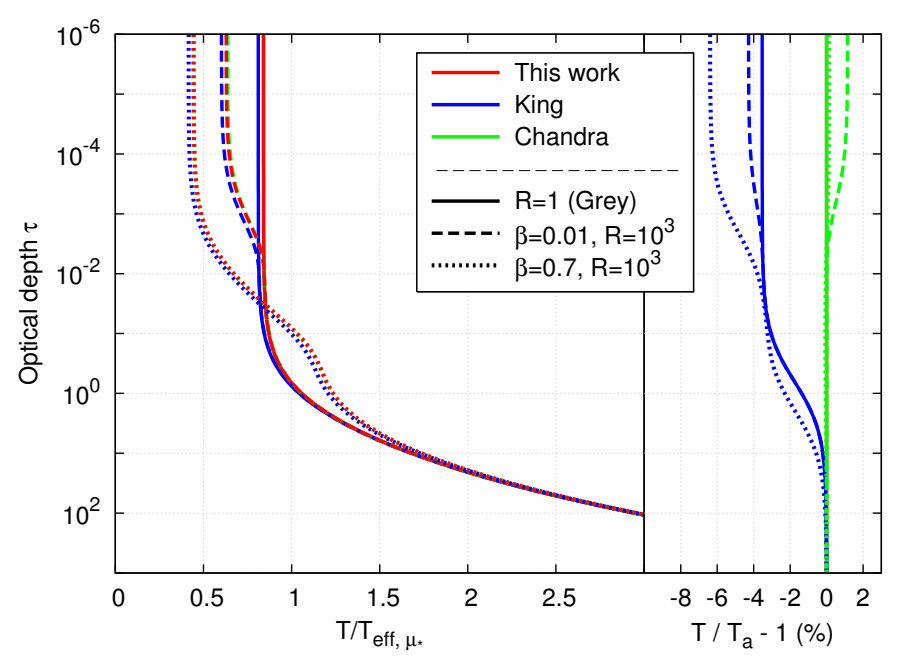

Fig. 4. Comparison of the non-irradiated solutions of the radiative transfer problem within the so-called picket-fence model approximation (see text). The left panel shows temperature (in $T_{\text {eff }}$ units) vs. optical depth. The right panel shows the relative temperature difference between our model and other works. The models shown correspond to the solutions of King (1955) (blue lines), Chandrasekhar (1935) (green lines), and this work (red lines). Different models correspond to the grey case (plain), i.e. $R=1$, and 2 non-grey cases: $\beta=0.01, R=10^{3}$ (dashed) and $\beta=0.7, R=10^{3}$ (dotted) where $R \equiv \kappa_{1} / \kappa_{2}$. The red and green lines are so similar that they are almost indistinguishable in the left panel.

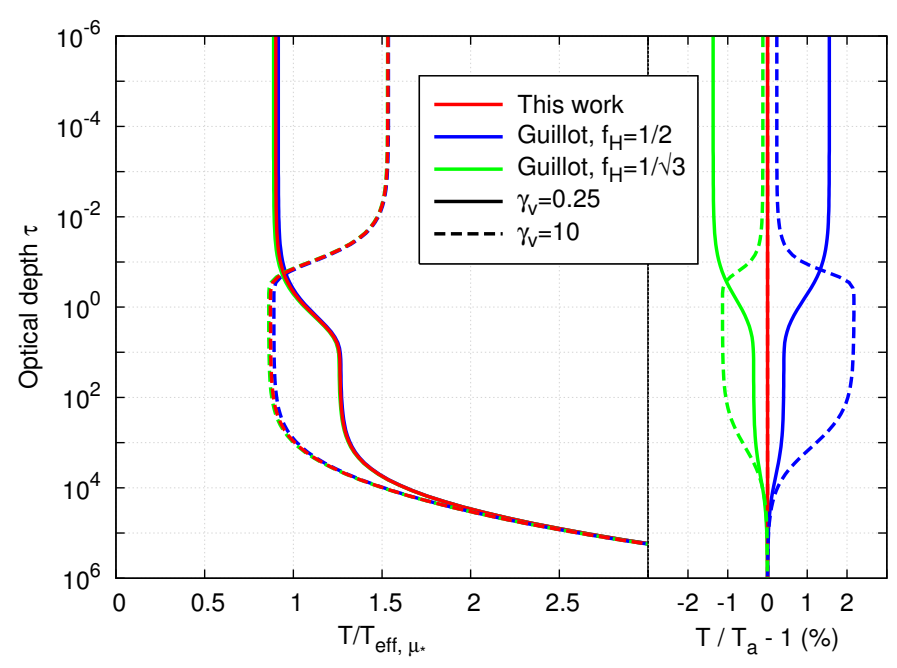

Fig. 5. Comparison between our model in the semi-grey limit and Guillot (2010). We used $\gamma_{\mathrm{v}}=0.25$ (plain line) and $\gamma_{\mathrm{v}}=10$ (dashed line). For the Guillot (2010) model we show the curves for two different boundary conditions: $f_{\mathrm{H}}=1 / 2$ (blue) and $f_{\mathrm{H}}=1 / \sqrt{3}$ (green). We used $\mu_{*}=1 / \sqrt{3}$.

to the approximation made on the angular dependency of the radiation field and implicitly linked to the choice of the different boundary conditions discussed in Sect. 2.3.2.

\subsection{Comparison of skin temperatures}

As discussed previously, the skin temperature (temperature at the limit of zero optical depth) is an important outcome of radiative transfer and in the case of non-irradiated models, an exact solution is available. We compare our results to other analytical results in Fig. 6. In the limit of a non-irradiated planet and in 
V. Parmentier and T. Guillot: A non-grey analytical model for irradiated atmospheres. I.

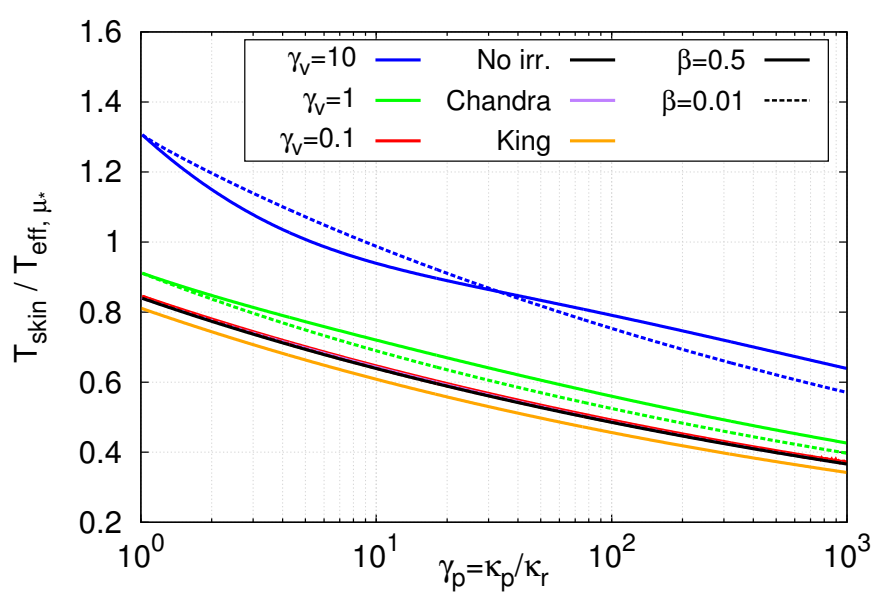

Fig. 6. Skin temperature of the planet given by our irradiated picketfence model for different values of $\gamma_{\mathrm{v}}$ and in the non-irradiated case. Curves for $\beta=0.01$ (plain lines) and $\beta=0.5$ (dashed lines) are shown. Skin temperature from Chandrasekhar (1935) and King (1956) are also shown. For the irradiated case we used $\mu_{*}=1 / \sqrt{3}$ and $T_{\mathrm{int}}=0$. The $\gamma_{\mathrm{v}}=0.1$, the non-irradiated, and the Chandrasekhar (1935) curves are closely packed.

the limit $\gamma_{\mathrm{v}}^{*} \rightarrow 0$, our skin temperature converges to the one derived by Chandrasekhar (1935). This is an important test for the model, as for low values of $\gamma_{\mathrm{v}}$, most of the stellar flux is absorbed in the deep layers of the planet and the model is expected to behave as a non-irradiated model with the same effective temperature. Moreover, we note that for low values of $\gamma_{\mathrm{v}}$, the skin temperature is affected only by $\gamma_{\mathrm{P}}$ as was already claimed by King (1956) and Chandrasekhar (1935). This conclusion no longer applies for higher values of $\gamma_{\mathrm{v}}$ for which the skin temperature also depends on $\beta$. This can be seen by comparing the dotted lines and plain lines of the same colour in Fig. 6. At a given value of $\gamma_{\mathrm{P}}$, a higher value of $\beta$ corresponds to a smaller $\kappa_{2} / \kappa_{1}$. Depending on the value of $\beta$, the stellar irradiation can be absorbed in a region which can be optically thick to the two thermal bands, only one, or none, leading to different behaviour for the skin temperature.

\section{Consequences of non-grey effects}

In this section we study the physical processes that shape our non-grey temperature profile. To overcome the apparent complexity of our solution, we first derive an approximate expression for the thermal fluxes at the top of the atmosphere. We then obtain a much simpler expression for the skin and the deep temperatures. Comparing these expressions with their semi-grey equivalent, we get physical insights into the processes that shape the temperature profile.

\subsection{Estimation of the fluxes in the different bands}

In steady state, all the energy that penetrates the atmosphere must be radiated away. Thus, the radiative equilibrium at the top of the atmosphere is of great importance to understand how the non-grey effects shape the temperature profile. In particular, whether the thermal fluxes are transported by the channel of highest opacity (channel 1) or the channel of lowest opacity (channel 2) is of particular importance.

As seen in Eq. (76), the contribution to the final temperature of the internal luminosity and of the external irradiation are independent. Thus, the thermal fluxes can be split into two independent contributions that can be studied separately:

$H_{i}=H_{i, \text { int }}+H_{i, \text { irr }}$.

Figure 7 shows which thermal band actually carries the thermal flux $H_{\text {irr }}(0)$ out of the atmosphere (the flux $F_{\text {irr }}(0)$ is equal to $\left.4 \pi H_{\text {irr }}(0)\right)$. This depends strongly on whether the stellar irradiation is absorbed in the upper or in the deep atmosphere. If it is deposited in the deep layers of the planet (i.e. $\gamma_{\mathrm{v}} \ll 1$ ), most of the flux is transported by the second thermal channel whatever the width of the second channel. Conversely, when the stellar irradiation is deposited in the upper atmosphere, most of the flux is carried by the first thermal channel whatever the width of the first channel. The tipping point, i.e. when each channel carries half of the flux, is reached when $\gamma_{\mathrm{v}}=\tau_{\text {lim }}^{-1}$. Figure 8 shows the variations of $\tau_{\lim }$ with the width and the strength of the two thermal opacity bands; $\tau_{\text {lim }}$ increases with $\beta$ but decreases with $R \equiv \kappa_{1} / \kappa_{2}$. It always corresponds to an optical depth where the first channel is optically thick and the second is optically thin.

For high values of $\gamma_{P}$ (i.e. $\gamma_{P}>2$ ), we can approximate the ratio of the thermal fluxes related to the irradiation by a much simpler expression:

$\frac{H_{1, \mathrm{irr}}(0)}{H_{2, \mathrm{irr}}(0)} \approx \frac{\beta}{\sqrt{\gamma_{\mathrm{P}}}}+\frac{1}{\frac{1-\beta}{\sqrt{\gamma_{\mathrm{P}}}}+\frac{1}{\gamma_{\mathrm{v}}^{*} \tau_{\lim }}}$.

As shown in Fig. 7 this expression correctly matches the expression of the analytical model. Depending on the value of $\gamma_{v}^{*} \tau_{\lim }$, the expression reduces to

$$
\begin{aligned}
& \frac{H_{1, \text { irr }}(0)}{H_{2, \text { irr }}(0)} \approx \frac{\beta}{\sqrt{\gamma_{\mathrm{P}}}}+\gamma_{\mathrm{v}}^{*} \tau_{\lim }, \text { when } \gamma_{\mathrm{v}}^{*} \tau_{\lim }<1 \\
& \frac{H_{2, \text { irr }}(0)}{H_{1, \text { irr }}(0)} \approx \frac{1-\beta}{\sqrt{\gamma_{\mathrm{P}}}}+\frac{1}{\gamma_{\mathrm{v}}^{*} \tau_{\lim }}, \text { when } \gamma_{\mathrm{v}}^{*} \tau_{\lim }>1 .
\end{aligned}
$$

We now look for a similar expression for the thermal fluxes resulting from the internal luminosity $\left(H_{\text {int }}\right)$. Because the internal luminosity irradiates the atmosphere from below, the resulting thermal fluxes behave similarly to the irradiated when $\gamma_{\mathrm{v}} \rightarrow 0$, thus we have

$$
\frac{H_{1, \text { int }}(0)}{H_{2, \text { int }}(0)} \approx \frac{\beta}{\sqrt{\gamma_{\mathrm{P}}}} \text {. }
$$

As $\gamma_{\mathrm{P}}$ is always greater than one and $\beta$ is always lower than one, the internal luminosity is always transported by channel 2 , the channel of lowest opacity.

\subsection{The skin temperature}

The skin temperature reveals the behaviour of the atmosphere at low optical depths. This is the part of the atmosphere probed during the transit of an exoplanet in front of its host star and is therefore of particular importance to interpret the observations. Figure 9 shows that in the irradiated case non-grey effects always tend to lower the skin temperature compared to the semi-grey case. This upper atmospheric cooling is already significant $(>10 \%)$ for slightly non-grey opacities (i.e. $\gamma_{\mathrm{P}} \approx 2$ ). For higher values of $\gamma_{\mathrm{P}}$ the cooling is stronger, reaching $50 \%$ for $\gamma_{\mathrm{P}} \approx 10-1000$. Conversely to the non-irradiated case, the skin temperature is not only a function of $\gamma_{\mathrm{P}}$ but also depends on $\beta$, i.e. not only are the mean opacities relevant, but also their actual shape. For high values of $\beta$, when the stellar irradiation is 


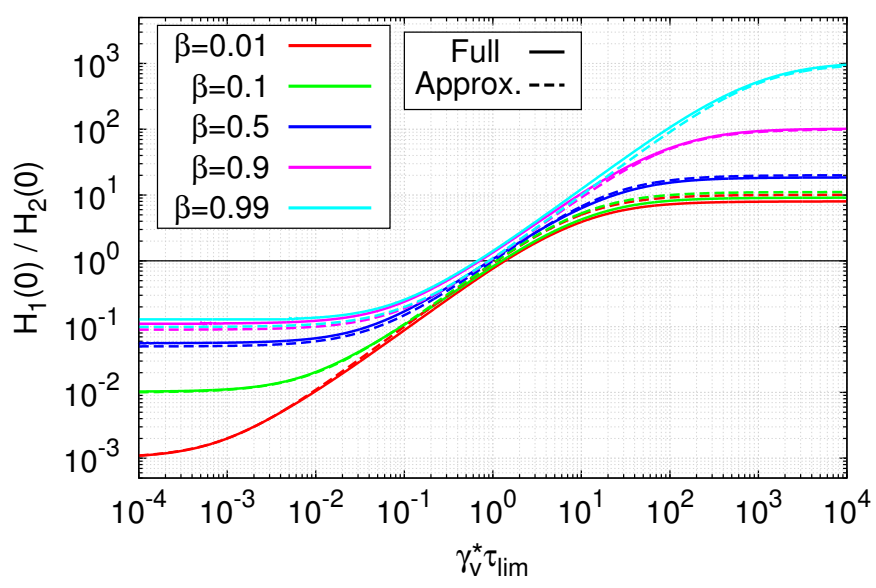

Fig. 7. Ratio of the total flux in the two thermal bands in function of $\gamma_{\mathrm{v}}^{*} \tau_{\lim }$ for different $\beta$ and for $\gamma_{\mathrm{P}}=100$ given by our analytical model (plain) and by Eq. (100) (dashed). We used $\mu_{*}=1 / \sqrt{3}$ and $T_{\mathrm{int}}=0$.

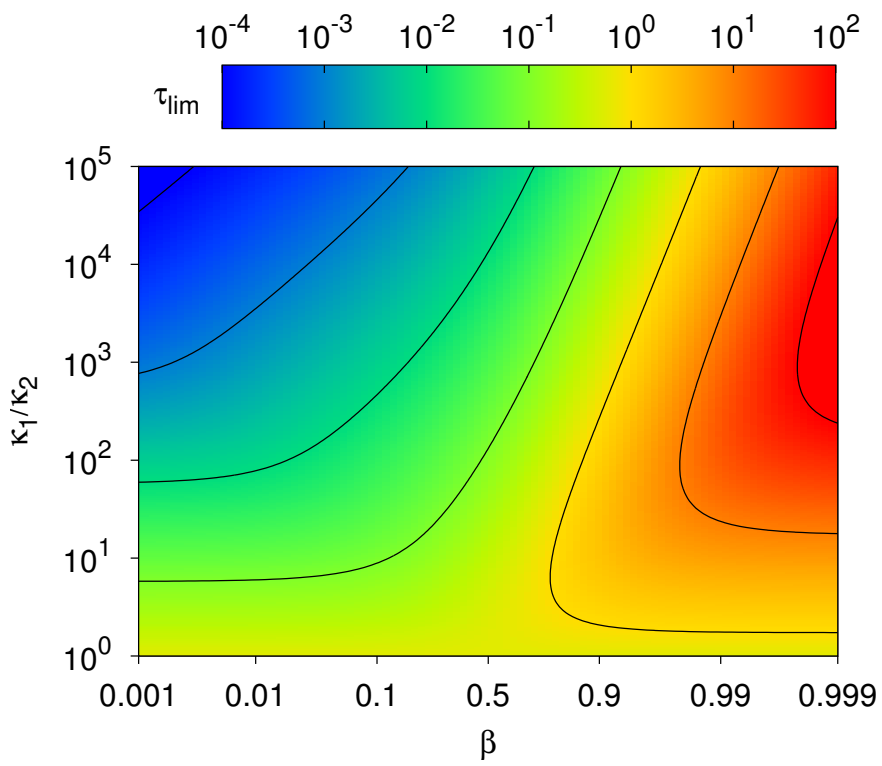

Fig. 8. Value of $\tau_{\text {lim }}$ in function of the width of the lines $\beta$ and their strength $\kappa_{1} / \kappa_{2}$. The $x$-axis is in logit scale, where the function logit is defined as $\operatorname{logit}(x)=\log (x /(1-x))$.

absorbed in the upper layers of the atmosphere (e.g. $\left.\gamma_{\mathrm{v}}=100\right)$ the cooling is more efficient than when the stellar irradiation is absorbed in the deep layers (e.g. $\gamma_{\mathrm{v}}=0.01$ ), whereas for low values of $\beta$ the cooling is independent of $\gamma_{\mathrm{v}}$.

The skin temperature results directly from the radiative equilibrium of the upper atmosphere. Using the boundary condition (49) in the radiative equlibrium Eq. (23) evaluated at $\tau=0$ we can write

$2 \gamma_{1} H_{1}(0)+2 \gamma_{2} H_{2}(0)-\gamma_{\mathrm{v}}^{*} H_{\mathrm{v}}(0)=\gamma_{\mathrm{P}} B(0)$,

where the skin temperature is given by $T_{\text {skin }}^{4}=\pi B(0) / \sigma$. The skin temperature, depends on the values of $H_{1}(0)$ and $H_{2}(0)$ and thus on whether the stellar irradiation is absorbed in the deep atmosphere or in the upper atmosphere.

\subsubsection{Case of deep absorption of the irradiation flux}

When $\gamma_{\mathrm{v}}^{*} \tau_{\text {lim }}<1$, the stellar irradiation is absorbed in the deep layers of the atmosphere, where the second thermal band, the band of lowest opacity, is optically thick. Thus, most of the flux is transported by the second thermal band and we have $H_{2}(0)=H_{\infty}-H_{\mathrm{v}}(0)$. For high values of $\gamma_{\mathrm{P}}$, using Eq. (101a) and Eq. (102) we get $\gamma_{1} H_{1}(0) / \gamma_{2} H_{2}(0)>\sqrt{\gamma_{\mathrm{P}}} /(1-\beta)$ which is always larger than one. Thus, although most of the flux is in the second thermal band, it is the first band, the band of highest opacity, that sets the radiative equilibrium. Neglecting the second term in Eq. (103) and calculating $H_{1}(0)$ with Eqs. (101a) and (102) we obtain:

$$
B(0)=\frac{2 \gamma_{1} \beta}{\gamma_{\mathrm{P}} \sqrt{\gamma_{\mathrm{P}}}} H_{\infty}-\left(\frac{2 \gamma_{1} \beta}{\gamma_{\mathrm{P}} \sqrt{\gamma_{\mathrm{P}}}}+\frac{2 \gamma_{\mathrm{v}}^{*} \tau_{\lim } \gamma_{1}}{\gamma_{\mathrm{P}}}+\frac{\gamma_{\mathrm{v}}^{*}}{\gamma_{\mathrm{P}}}\right) H_{\mathrm{v}}(0) .
$$

Noting that for high values of $\gamma_{\mathrm{P}}, \tau_{\lim } \approx \beta(1-\beta)^{-1}\left(\sqrt{3 \gamma_{\mathrm{P}}}\right)^{-1}$, and $\gamma_{1} \approx \gamma_{\mathrm{P}} / \beta$, the equation becomes

$B(0)=\frac{2}{\sqrt{\gamma_{\mathrm{P}}}} H_{\infty}-\left(\frac{2}{\sqrt{\gamma_{\mathrm{P}}}}+\frac{2 \gamma_{\mathrm{v}}^{*}}{(1-\beta) \sqrt{3 \gamma_{\mathrm{P}}}}+\frac{\gamma_{\mathrm{v}}^{*}}{\gamma_{\mathrm{P}}}\right) H_{\mathrm{v}}(0)$.

Replacing the fluxes by their equivalent temperature we get an expression for the skin temperature valid for $\gamma_{\mathrm{v}}^{*} \tau_{\text {lim }}<1$ and $\gamma_{\mathrm{P}}>2$ :

$T_{\text {skin }}^{4}=\frac{2}{\sqrt{\gamma_{\mathrm{P}}}} \frac{T_{\mathrm{int}}^{4}+\mu_{*} T_{\mathrm{irr}}^{4}}{4}+\left(\frac{2 \gamma_{\mathrm{v}}^{*}}{(1-\beta) \sqrt{3 \gamma_{\mathrm{P}}}}+\frac{\gamma_{\mathrm{v}}^{*}}{\gamma_{\mathrm{P}}}\right) \frac{\mu_{*} T_{\mathrm{irr}}^{4}}{4}$.

When $\gamma_{\mathrm{v}}^{*} \tau_{\lim }<1$, the first term dominates and the expression differs by a factor of $1 / \sqrt{\gamma_{\mathrm{P}}}$ from the semi-grey case (Eq. (31)). Because $\gamma_{\mathrm{P}}>1$ for non-grey opacities, the skin temperature is always smaller in the non-grey case than in the grey case, as shown in Fig. 9.

Physical interpretation. When $\gamma_{\mathrm{v}}^{*} \tau_{\lim }<1$ most of the irradiation is absorbed where both thermal channels are optically thick. The flux is mainly transported by the channel of lowest opacity $\kappa_{2}$ but only the residual flux transported by the channel of highest opacity $\kappa_{1}$ contributes to the radiative equilibrium at the top of the atmosphere. Because it represents only a small part of the total flux, the upper atmosphere does not need to radiate a lot of energy and thus the upper atmospheric temperatures are smaller than in the semi-grey case. The larger the departure from the semi-grey opacities, the cooler the skin temperature, without lower bounds.

\subsubsection{Case of shallow absorption of the irradiation flux}

When $\gamma_{\mathrm{v}}^{*} \tau_{\text {lim }}>1$, most of the stellar irradiation is absorbed in the upper atmosphere, where only the first thermal band is optically thick. According to Eq. (101a), most of the flux originating from the irradiation $H_{\text {irr }}(0)$ is carried by the first thermal band, the band of highest opacity. Conversely, following Eq. (102), the internal luminosity is still transported by the second thermal channel, as in the $\gamma_{\mathrm{v}} \tau_{\lim }<1$ case. As $\gamma_{1}>\gamma_{2}$, the radiative equilibrium of the upper atmosphere is still determined by the channel of highest opacity, channel 1 , and the second term of Eq. (103) can be neglected. Conversely to the case $\gamma_{\mathrm{v}} \tau_{\lim }<1$, the top boundary condition now reads $H_{1}(0) \approx H_{1}$, int $-H_{\mathrm{v}}(0)$. Using Eq. (102) to calculate $H_{1 \text {, int }}$ and noting that for high values of $\gamma_{\mathrm{P}}, \gamma_{\mathrm{P}} \approx \beta \gamma_{1}$, the radiative equilibrium becomes

$B(0)=\frac{2}{\sqrt{\gamma_{\mathrm{P}}}} H_{\infty}-\left(\frac{2}{\beta}+\frac{\gamma_{\mathrm{v}}^{*}}{\gamma_{\mathrm{P}}}\right) H_{\mathrm{v}}(0)$. 
V. Parmentier and T. Guillot: A non-grey analytical model for irradiated atmospheres. I.

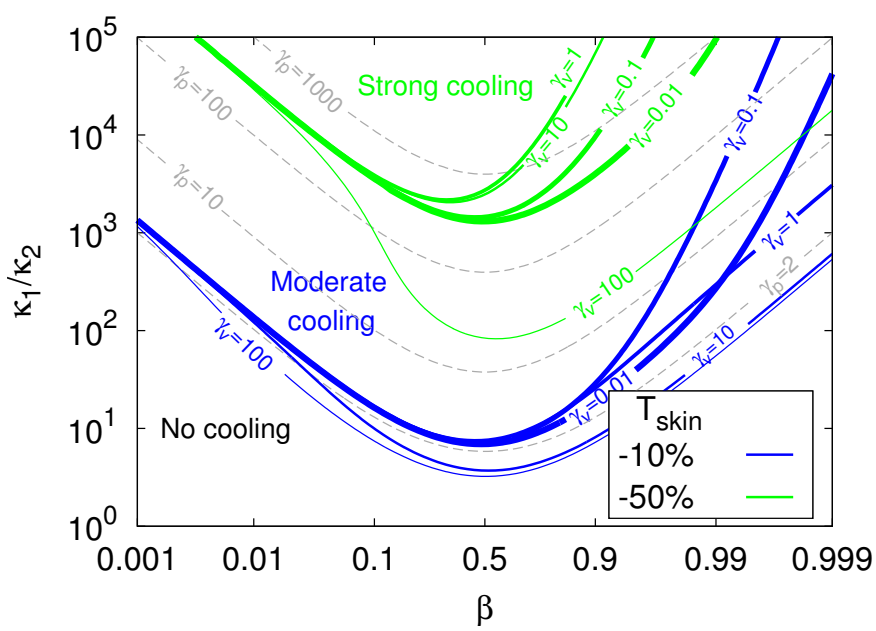

Fig. 9. Contours of the relative difference between the skin temperature in the non-grey model and in the semi-grey model for different values of $\gamma_{\mathrm{v}}$ in function of the width of the lines and their strength. The non-grey atmosphere is $10 \%$ (resp. 50\%) cooler than the semi-grey atmosphere above the blue (resp. green) lines. The dashed lines are contours of $\gamma_{\mathrm{P}}$. We used $\mu_{*}=1 / \sqrt{3}$.

Replacing the fluxes by their equivalent temperatures we get an expression for the skin temperature valid for $\gamma_{\mathrm{v}}^{*} \tau_{\lim }>1$ and $\gamma_{\mathrm{P}}>2$

$T_{\text {skin }}^{4}=\frac{2}{\sqrt{\gamma_{\mathrm{P}}}} \frac{T_{\mathrm{int}}^{4}}{4}+\left(\frac{2}{\beta}+\frac{\gamma_{\mathrm{v}}^{*}}{\gamma_{\mathrm{P}}}\right) \frac{\mu_{*} T_{\mathrm{irr}}^{4}}{4}$.

This relation differs from the case $\gamma_{\mathrm{v}}^{*} \tau_{\lim }<1$ as the factor $1 / \sqrt{\gamma_{\mathrm{P}}}$ before the irradiation temperatures is replaced by a factor $1 / \beta$. Thus, the skin temperature no longer becomes arbitrarily low. However, for high values of $\gamma_{v}$, the second term in the parenthesis dominates and the skin temperature decreases proportionally to $1 / \sqrt{\gamma_{\mathrm{P}}}$, which is faster than in the case $\gamma_{\mathrm{v}}^{*} \tau_{\lim }<1$. As an example, in Fig. 9, for $\gamma_{\mathrm{v}}=100$, the skin temperature decreases much faster when $\gamma_{\mathrm{P}}$ increases for large values of $\beta$ (i.e. when $\gamma_{\mathrm{v}}^{*} \tau_{\text {lim }}>1$ ).

Physical interpretation. When $\gamma_{\mathrm{v}}^{*} \tau_{\lim }>1$, most of the incident irradiation is absorbed in the upper atmosphere, where the second channel is optically thin. Therefore it is mainly transported by the channel of highest opacity: channel 1. Similarly to the case $\gamma_{\mathrm{v}}^{*} \tau_{\lim }<1$, the radiative equilibrium at the top of the atmosphere is set by the channel of highest opacity, the one that carries most of the thermal flux. Therefore all the flux from the irradiation contributes to the radiative equilibrium of the upper layers and the skin temperature cannot cool as much as in the $\gamma_{\mathrm{v}}^{*} \tau_{\lim }<1$ case, its lowest value being $\mu^{*} T_{\mathrm{irr}}^{4} / 2 \beta$. However, for high values of $\gamma_{\mathrm{v}}^{*}$ and as long as $\gamma_{\mathrm{P}}<\gamma_{\mathrm{v}}^{*}, T_{\text {skin }}^{4}$ decreases faster than in the case $\gamma_{\mathrm{v}}^{*} \tau_{\lim }<1$. This confines the stratosphere due to $\gamma_{\mathrm{v}}^{*}>1$ (i.e. the atmospheric levels with a temperature inversion) around the $\tau=\tau_{\text {lim }}$ level whereas it extends up to $\tau=0$ in the semi-grey case (see Figs. 12-14 hereafter).

\subsection{The deep temperature}

The temperature of the deep atmosphere is a fundamental outcome from radiative transfer models as it reveals the energy exchange between the planet and its surroundings. Therefore, it is often used as a boundary condition of planetary interior models.

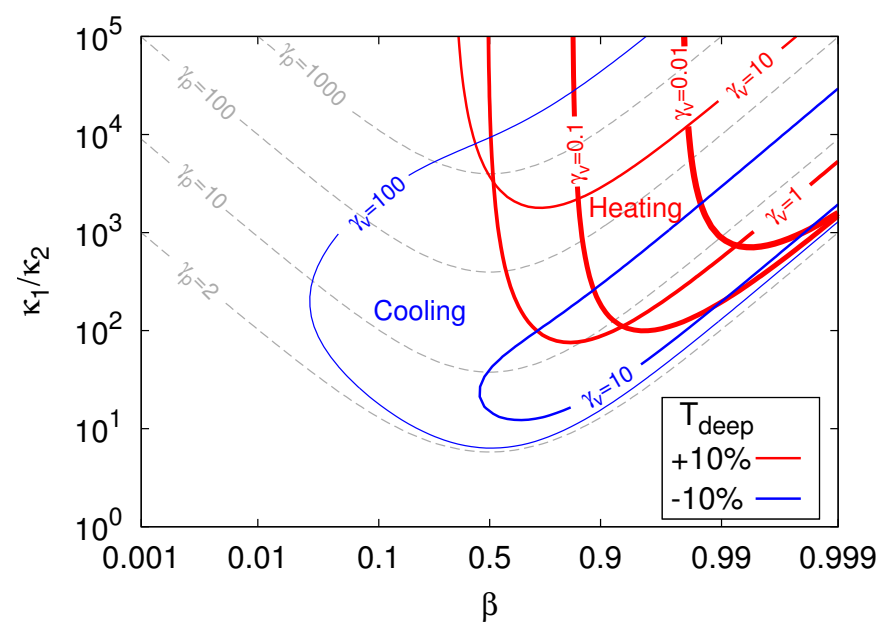

Fig. 10. Contours of the relative difference between the deep temperature in the non-grey model and in the semi-grey model for different values of $\gamma_{v}$ in function of the width of the lines and their strength. The non-grey atmosphere is $10 \%$ hotter (resp. cooler) than the semi-grey atmosphere inside the red (resp. blue) contours. The dashed lines are contours of $\gamma_{\mathrm{P}}$. We used $\mu_{*}=1 / \sqrt{3}$.

We define the deep temperature as

$T_{\text {deep }}^{4}=\lim _{\tau \rightarrow \infty} T(\tau)^{4}-3 T_{\text {int }}^{4} \tau$.

Thus, the temperature of the deep atmosphere can be approximated as $T(\tau)^{4}=T_{\text {deep }}^{4}+3 T_{\text {int }}^{4} \tau$ between the $\tau \approx 1$ level and the radiative/convective boundary. For irradiated planets, the deep temperature corresponds to the isothermal zone around $\tau \approx 1$ and is close to the temperature at 10 bar often used as a boundary condition for interior models (e.g., Burrows et al. 1997; Guillot \& Showman 2002). As seen in Fig. 10, the deep temperature has a complex behaviour. For low values of $\gamma_{\mathrm{v}}$, whenever $\beta$ becomes large enough, the deep temperature increases compared to the semi-grey case, an effect known as the line blanketing effect in the stellar literature (e.g. Milne 1921; Chandrasekhar 1935; Hubeny \& Lanz 1995). This effect is always maximum when $\gamma_{\mathrm{v}}^{*} \approx \tau_{\text {lim }}^{-1}$ (see Fig. 8). Conversely, for high values of $\gamma_{\mathrm{v}}^{*}$ (i.e. $\gamma_{\mathrm{v}}^{*}>10$ ), the deep atmosphere warms up only for high values of $\gamma_{\mathrm{P}}\left(\gamma_{\mathrm{P}}>\gamma_{\mathrm{v}}^{2}\right)$ whereas it becomes cooler than in the semi-grey case for lower values of $\gamma_{\mathrm{P}}$, a behaviour that was not spotted in previous analytical models.

The deep atmospheric temperature is directly set by the boundary condition at the top of the atmosphere. From Eq. (58), we see that when $\tau \rightarrow \infty$,

$T_{\text {deep }}^{4}=\lim _{\tau \rightarrow \infty} B(\tau)-3 H_{\infty} \tau=C_{1}$,

where $C_{1}$ is set by the top boundary condition (49) applied on $J_{\gamma}(0)$ (see Eq. (52)):

$2 \frac{H_{1}(0)}{\gamma_{1}}+2 \frac{H_{2}(0)}{\gamma_{2}}=C_{1}+\frac{3}{\gamma_{\mathrm{v}}^{*}} H_{\mathrm{v}}$.

Similarly to the skin temperature, the deep temperature depends on $H_{1}(0)$ and $H_{2}(0)$, and also depends on whether the thermal flux is transported by the first or by the second thermal channel, i.e. whether $\gamma_{\mathrm{v}} \tau_{\text {lim }}$ is larger or smaller than one. 


\subsubsection{Case of deep absorption of the irradiation flux}

In the case $\gamma_{\mathrm{v}}^{*} \tau_{\lim }<1$, most of the thermal flux is transported by the second thermal channel and because $\gamma_{1} \gg \gamma_{2}$ we can write

$2 \frac{H_{2}(0)}{\gamma_{2}} \approx C_{1}+\frac{3}{\gamma_{\mathrm{v}}^{*}} H_{\mathrm{v}}$,

applying the radiative equilibrium at the top of the atmosphere, and considering that most of the flux is carried by the second thermal channel, we get

$H_{2}(0) \approx H_{\infty}-H_{\mathrm{v}}(0)$.

Thus, we can calculate $C_{1}$ and obtain

$B(\tau)-3 H_{\infty} \tau \underset{\tau \rightarrow \infty}{\sim} \frac{2}{\gamma_{2}} H_{\infty}-\left(\frac{2}{\gamma_{2}}+\frac{3}{\gamma_{\mathrm{v}}^{*}}\right) H_{\mathrm{v}}(0)$.

For high values of $\gamma_{\mathrm{P}}, \gamma_{2} \approx(1-\beta)$. Replacing the fluxes by their equivalent temperatures we get an expression for $T_{\text {deep }}$ valid for $\gamma_{\mathrm{v}}^{*} \tau_{\lim }<1$ and $\gamma_{\mathrm{P}}>2$ :

$T_{\text {deep }}^{4} \approx \frac{2}{1-\beta} \frac{T_{\mathrm{int}}^{4}+\mu_{*} T_{\mathrm{irr}}^{4}}{4}+\frac{3}{\gamma_{\mathrm{v}}^{*}} \frac{\mu_{*} T_{\mathrm{irr}}^{4}}{4}$.

This expression differs from the semi-grey value of Guillot (2010) by a factor $1 /(1-\beta)$ multiplying the first term. Thus, when $\beta \rightarrow 1$, the temperature becomes warmer than in the semi-grey case, as seen for the low values of $\gamma_{\mathrm{v}}$ in Fig. 10.

Physical interpretation. When $\gamma_{\mathrm{v}}^{*} \tau_{\lim }<1$, most of the flux from the star is absorbed in the deep atmosphere and is principally transported by the channel of lowest opacity (channel 2), even when the width of this channel is smaller than the width of the first thermal channel. Whenever $\beta \rightarrow 1$, the width of the second channel decreases. In order to keep transporting most of the thermal flux, the flux per wavelength in the second channel must increase. This increases the temperature where the second channel is optically thick, i.e. in the deep atmosphere. This is equivalent to the line blanketing effect that has been well studied in stars (see Milne 1921; Chandrasekhar 1935; Hubeny \& Lanz 1995, for example).

\subsubsection{Case of shallow absorption of the irradiation flux}

When $\gamma_{\mathrm{v}}^{*} \tau_{\lim }>1$ most of the irradiation flux is absorbed in a region where the second thermal channel is optically thin. Thus the flux is carried by the first thermal channel and we have $H_{1, \text { irr }}(0) \gg H_{2 \text {,irr }}(0)$. However, because $\gamma_{2} \ll \gamma_{1}$, we can use Eq. (101b) to show that $\left(H_{2, \text { irr }}(0) / \gamma_{2}\right) /\left(H_{1, \text { irr }}(0) / \gamma_{1}\right)>\sqrt{\gamma_{\mathrm{P}}} / \beta$, which is larger than 1 . Thus Eq. (112) remains valid. However, conversely to the case $\gamma_{\mathrm{v}}^{*} \tau_{\lim }<1$, the top boundary condition now reads

$H_{1}(0) \approx-H_{\mathrm{v}}(0)+H_{2, \text { int }}$

$\mathrm{H}_{2}(0) \approx \mathrm{H}_{\infty}+\mathrm{H}_{2, \mathrm{irr}}$,

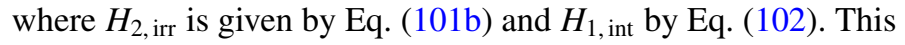
leads to:

$C_{1}=\frac{2}{\gamma_{2}} \frac{1-\beta}{\sqrt{\gamma_{\mathrm{P}}}}\left(H_{\infty}-H_{\mathrm{v}}(0)\right)-\frac{2}{\gamma_{2} \gamma_{\mathrm{v}}^{*} \tau_{\lim }} H_{\mathrm{v}}(0)-\frac{3}{\gamma_{\mathrm{v}}} H_{\mathrm{v}}(0)$.

Again, for large values of $\gamma_{\mathrm{P}}, \gamma_{2} \rightarrow 1-\beta$ and replacing the fluxes by their equivalent temperatures we get an expression for $T_{\text {deep }}$ valid for $\gamma_{\mathrm{V}}^{*} \tau_{\lim }>1$ and $\gamma_{\mathrm{P}}>2$ :

$T_{\text {deep }}^{4}=\frac{2}{1-\beta} \frac{T_{\text {int }}^{4}}{4}+\frac{2}{\sqrt{\gamma_{\mathrm{P}}}} \frac{\mu^{*} T_{\mathrm{irr}}^{4}}{4}+\left(\frac{3}{\gamma_{\mathrm{v}}}+\frac{2}{1-\beta} \frac{1}{\gamma_{\mathrm{v}}^{*} \tau_{\lim }}\right) \frac{\mu^{*} T_{\mathrm{irr}}^{4}}{4}$.

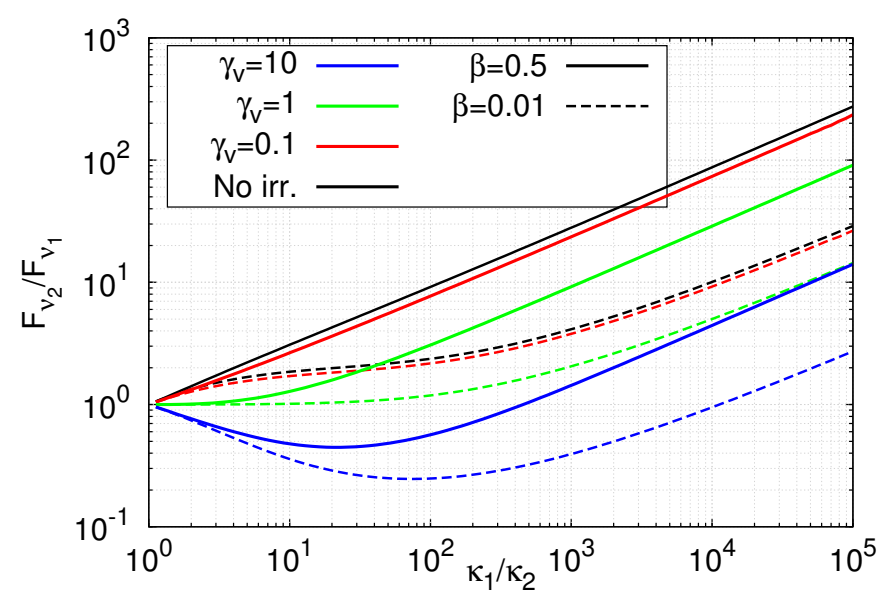

Fig. 11. Ratio of the monochromatic flux in the two bands $F_{v_{2}} / F_{v_{1}}=$ $\beta H_{2}(0) /(1-\beta) H_{1}(0)$ in function of the opacity ratio $\kappa_{1} / \kappa_{2}$ for different bandwidths $\beta$ and visible to infrared opacities $\gamma_{\mathrm{v}}$. We used $\mu_{*}=1 / \sqrt{3}$.

When $\gamma_{v}^{*} \tau_{\text {lim }} \gg 1$, the contribution to the deep temperature of the irradiation temperature becomes inversely proportional to $\sqrt{\gamma_{\mathrm{P}}}$. As $\gamma_{\mathrm{P}}>1$, the deep temperature is smaller in the non-grey case than in the semi-grey case. This is illustrated by the cases $\gamma_{\mathrm{v}}=10$ and $\gamma_{\mathrm{v}}=100$ in Fig. 10. When $\gamma_{\mathrm{v}}^{*} \tau_{\lim } \rightarrow 1$, the term in $1 / \sqrt{\gamma_{\mathrm{P}}}$ becomes very small compared to the term in $1 /(1-\beta)$ and the expression converges toward equation Eq. (115), valid for $\gamma_{\mathrm{v}}^{*} \tau_{\lim }<1$.

Physical interpretation. When $\gamma_{\mathrm{v}}^{*} \tau_{\lim } \gg 1$, the incident irradiation is absorbed in the upper atmosphere, where only the channel of highest opacity is optically thick. Thus, the channel of highest opacity $\kappa_{1}$ transports all the energy and radiates it directly to space. The incident irradiation is no more transported to the deep atmosphere, leading to a cooler deep atmosphere.

\subsection{Outgoing flux}

During secondary eclipse observations, the flux emitted by the planet can be observed in different bands (e.g. Seager \& Deming 2010). The detection of molecular species in the emission spectrum of an exoplanet depends strongly on the flux contrast between the continuum and the molecular band considered, which in turn depends on the temperature profile. Figure 11 shows the flux per wavelength emitted in the first band $\left(F_{v_{1}}=4 \pi H_{1}(0) / \beta\right)$ over the flux per wavelength emitted in the second band $\left(F_{v_{2}}=\right.$ $\left.4 \pi H_{2}(0) /(1-\beta)\right)$. This would be the expected contrast in the emission spectrum of the planet between the spectral features and the continuum. For a non-irradiated atmosphere and for low values of $\gamma_{\mathrm{v}}$ this is a monotonic function of the opacity ratio $\kappa_{1} / \kappa_{2}$. The flux in the band of lowest opacity is always bigger than the flux in the band of highest opacity, i.e. we see absorption bands. For large values of $\gamma_{\mathrm{v}}$, whenever a strong temperature inversion happens the absorption bands turn into emission bands. Those different behaviours are captured by the simple expression (100). Note that in all cases, for large values of $\kappa_{1} / \kappa_{2}$ we have:

$\frac{F_{v_{2}}}{F_{v_{1}}} \propto\left(\frac{\kappa_{1}}{\kappa_{2}}\right)^{1 / 2}$ 
V. Parmentier and T. Guillot: A non-grey analytical model for irradiated atmospheres. I.

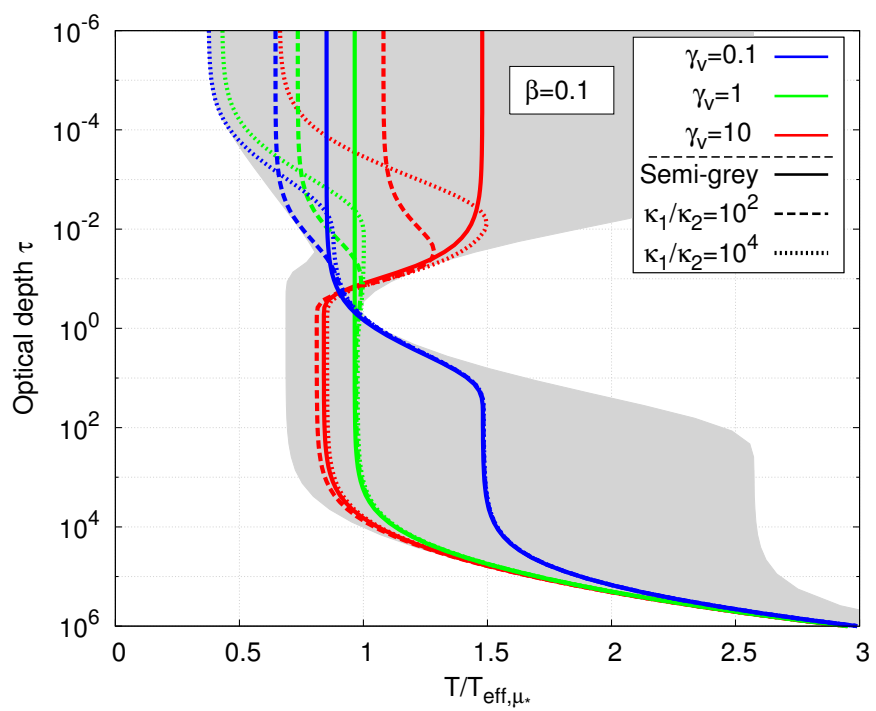

Fig. 12. Pressure/temperature profiles for an irradiated planet $\left(T_{\mathrm{int}}=\right.$ $T_{\text {irr }} / 10$ and $\mu_{*}=1 / \sqrt{3}$ ). The shaded area shows the full range of parameters $10^{-3}<\beta<10^{-1}, 1<R<10^{4}$ and $0.01<\gamma_{\mathrm{v}}<100$. The lines are profiles obtained for $\beta=0.1$; for $R=1$ (plain lines), $R=100$ (dashed lines), and $R=10^{4}$ (dotted lines); and for $\gamma_{\mathrm{v}}=0.1$ (blue), $\gamma_{\mathrm{v}}=1$ (green), and $\gamma_{\mathrm{v}}=10$ (red).

\section{Resulting temperature profiles}

No matter how strong the non-greyness of the opacities is, there is always a region, at high enough optical depth, where the nongrey solution converges toward the grey solution (see e.g. Fig. 4). The transition between a regime where the grey model is accurate to a regime where the non-grey effects are of prime importance is set by the parameter $\tau_{\text {lim }}$. For optical depths lower than $\tau_{\text {lim }}$, non-grey effects are always important, whereas for optical depths higher than $\tau_{\text {lim }}$, non-grey effects are present only if $\gamma_{\mathrm{v}} \tau_{\lim }<1$ and $\beta \rightarrow 1$. Three distinct situations can be observed in Fig. 8. For narrow lines $(\beta<0.1), \tau_{\lim }$ is always smaller than one, for larger lines or molecular bands $(0.1<\beta<0.9), \tau_{\lim }$ is close to one, whereas for inverted lines $(0.9<\beta<1), \tau_{\text {lim }}$ can reach much higher values. Thus, when $\gamma_{\mathrm{v}} \gg 1$, few non-grey effects are expected in the deep atmosphere, contrary to the cases $\gamma_{\mathrm{v}} \approx 1$ and $\gamma_{\mathrm{v}} \ll 1$.

In the case of narrow lines $(\beta<0.1)$, only the non-grey cooling of the upper atmosphere is effective. As shown in Fig. 12, the profile remains close to the semi-grey model at large optical depths. However, at low optical depths, for $\tau<\tau_{\text {lim }}$, the atmosphere can be much cooler than in the semi-grey case (case $R=1$ ). In particular, in the $\gamma_{\mathrm{v}}=10$ case, the non-grey cooling localizes the temperature inversion to a specific layer, contrary to the semi-grey case where it extends to the top of the atmosphere. The envelope of all the profiles (shaded area) is much wider than in the semi-grey case (see Fig. 1).

In the case of large lines or molecular bands $(0.1<\beta<0.9)$ shown in Fig. 13, both the non-grey cooling of the upper atmosphere and the blanketing effect are important. Whereas the upper atmosphere undergoes an efficient cooling, the lower atmosphere $(\tau>1)$ can experience a significant warming via the blanketing effect. Lowering the ability of the deep atmosphere to cool down efficiently can significantly affect the evolution of the planet (Parmentier \& Guillot 2011; Budaj et al. 2012; Spiegel \& Burrows 2013; Rauscher \& Showman 2013) and could contribute to the radius anomaly of hot-Jupiters (e.g. Guillot \& Showman 2002; Laughlin et al. 2011). Whenever $\gamma_{\mathrm{v}}^{*} \tau_{\lim } \approx 1$,

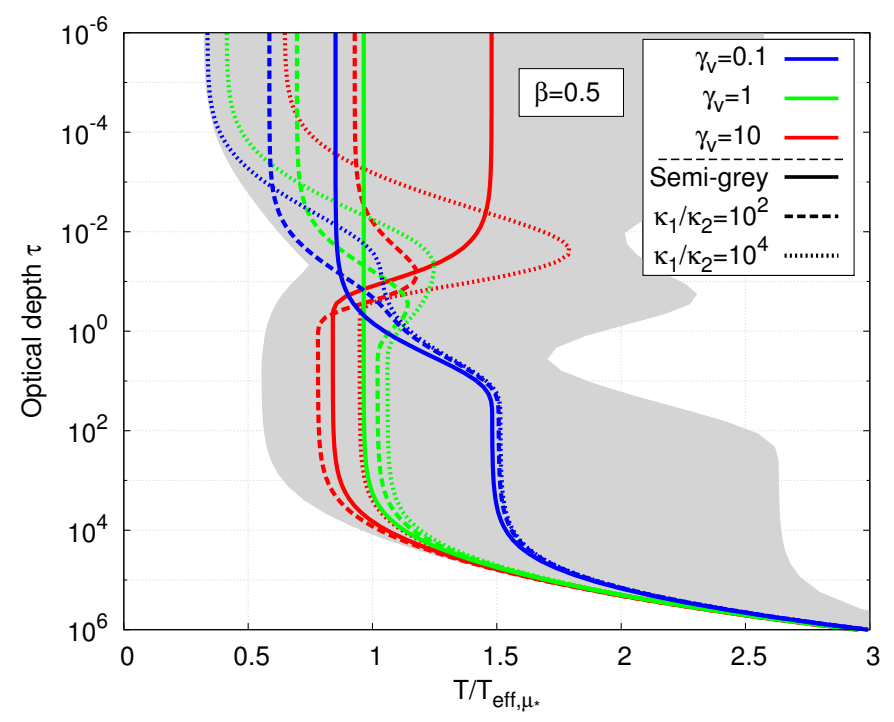

Fig. 13. Pressure/temperature profiles for an irradiated planet $\left(T_{\mathrm{int}}=\right.$ $T_{\text {irr }} / 10$ and $\left.\mu_{*}=1 / \sqrt{3}\right)$. The shaded area shows the full range of parameters $0.1<\beta<0.9,1<R<10^{4}$ and $0.01<\gamma_{\mathrm{v}}<100$. The lines are profiles obtained for $\beta=0.5$; for $R=1$ (plain lines), $R=100$ (dashed lines), and $R=10^{4}$ (dotted lines); and for $\gamma_{\mathrm{v}}=0.1$ (blue), $\gamma_{\mathrm{v}}=1$ (green), and $\gamma_{\mathrm{v}}=10$ (red).

the stellar irradiation is deposited at a level where non-grey effects lower the ability of the atmosphere to cool down efficiently. This leads to an efficient and localized warming causing a temperature inversion in the profile at $\tau \approx 1 / \gamma_{\mathrm{v}}^{*}$, even when none is expected from the semi-grey model (i.e. even when $\gamma_{\mathrm{v}}^{*}<1$ ). This happens, for example, when $\beta \approx 0.5$ for $\gamma_{\mathrm{v}}=10$, when $\beta \approx 0.9$ for $\gamma_{\mathrm{v}}=1$, and for $\beta \approx 0.99$ for $\gamma_{\mathrm{v}}=0.1$ (see Fig. 14).

In the case of inverted lines $(\beta>0.9)$, shown in Fig. 14, both the upper temperature and the deep temperature are affected by the non-grey effects. The upper atmosphere cools significantly compared to the semi-grey case. The deep atmosphere can either warm up because of the blanketing effect but, for high values of $\gamma_{\mathrm{v}}$ it can also become cooler than in the semi-grey case (see the case $\gamma_{\mathrm{v}}=10$ and $R=100$ in Fig. 14). Temperatures as cool as $0.5 T_{\text {eff, } \mu_{*}}$ can be reached. This is fundamentally different from the semi-grey case where the deep temperature is always larger than $2^{1 / 4} T_{\text {eff, } \mu_{*}}$ (see Fig. 3).

As $\beta$ increases, $\tau_{\text {lim }}$ increases and the blanketing effect disappears. Eventually, when $\beta \rightarrow 1$, the opacities, and thus the profile, become semi-grey again.

In summary, our irradiated picket-fence model can reach the whole temperature range span by the numerical models (see the shaded area in Figs. 12 to 14). Our model should therefore be preferred to classical semi-grey models as an approximate solution for the temperature profile of irradiated atmospheres.

\section{Conclusion}

We derived an analytic non-grey model to approximate the structure of a plane-parallel irradiated planetary atmosphere. Our model includes both thermal and visible non-grey opacities. The thermal and visible opacities are in the form of a two different picket-fence opacity functions. the thermal opacities are parametrized by the ratio of the visible to the infrared Rosseland mean opacities $\left(\gamma_{\mathrm{v}}\right)$, the ratio of the Planck to the Rosseland mean thermal opacities $\left(\gamma_{\mathrm{P}}\right)$, and the spectral width of the lines $(\beta)$. The model is valid for any functional form of the 
Table 2. Main quantities used in this paper.

\begin{tabular}{|c|c|c|c|}
\hline Symbol & Quantity & Definition & Units \\
\hline$\kappa_{\mathrm{R}}$ & Rosseland mean opacities & Eq. (2) & $\mathrm{m}^{2} / \mathrm{kg}$ \\
\hline$\kappa_{\mathrm{P}}$ & Planck mean opacities & Eq. (5) & $\mathrm{m}^{2} / \mathrm{kg}$ \\
\hline$\kappa_{1}$ & Opacity in the first band & Eq. (8) & $\mathrm{m}^{2} / \mathrm{kg}$ \\
\hline $\begin{array}{ll}\kappa_{1} \\
\kappa_{2}\end{array}$ & Opacity in the second band & Eq. (8) & $\mathrm{m}^{2} / \mathrm{kg}$ \\
\hline$\beta$ & Relative width of the first band & Eq. (9) & - \\
\hline$\kappa_{\mathrm{v}}$ & Opacity in the visible & Eq. (7) & $\mathrm{m}^{2} / \mathrm{kg}$ \\
\hline$\tau$ & Rosseland optical depth & Eq. (3) & - \\
\hline$R$ & Opacity ratio $\kappa_{1} / \kappa_{2}$ & Sect.3.4 & - \\
\hline$\gamma_{1}, \gamma_{2}, \gamma_{\mathrm{P}}, \gamma_{\mathrm{v}}$ & $\kappa_{1} / \kappa_{\mathrm{R}}, \kappa_{2} / \kappa_{\mathrm{R}}, \kappa_{\mathrm{P}} / \kappa_{\mathrm{R}}, \kappa_{\mathrm{v}} / \kappa_{\mathrm{R}}$ & Eqs. (12), (13), (6) and (7) & - \\
\hline$\tau_{\lim }$ & Limit optical depth & Eq. (15) & $\mathrm{Hz}$ \\
\hline$v$ & Frequency & - & $\mathrm{Hz}$ \\
\hline$\mu$ & cosine of the direction angle $\theta$ & - & - \\
\hline$\mu_{*}$ & cosine of the angle between the vertical and the star & - & - \\
\hline$\gamma_{\mathrm{v}}^{*}$ & $\gamma_{\mathrm{v}} / \mu_{*}$ & - & - \\
\hline$I_{\mu v}$ & Specific intensity at frequency $v$ and in the direction $\mu$ & Chandrasekhar (1960) & $\mathrm{W} / \mathrm{m}^{2} / \mathrm{Hz} / \mathrm{sr}$ \\
\hline$J_{v}, H_{v}, K_{v}$ & First, second, and third momentum of the specific intensity & Eq. $(20)$ & $\mathrm{W} / \mathrm{m}^{2} / \mathrm{Hz}$ \\
\hline$J, H, K$ & Integrated values of $J_{v}, H_{v}$, and $K_{v}$ & - & $\mathrm{W} / \mathrm{m}^{2}$ \\
\hline $4 \pi H_{\infty}=\sigma T_{\text {int }}^{4}$ & Internal flux from the planet & - & $\mathrm{W} / \mathrm{m}^{2}$ \\
\hline$\sigma T_{\text {sub }}^{4}-\sigma T_{\text {int }}$ & Stellar flux arriving at the substellar point of the planet & Sect. 3.7 & $\mathrm{~W} / \mathrm{m}^{2}$ \\
\hline $4 \pi H_{\mathrm{v}}(0)=-\sigma \mu_{*} T_{\mathrm{irr}}^{4}$ & Stellar flux that penetrates the modelled atmospheric column & - & $\mathrm{W} / \mathrm{m}^{2}$ \\
\hline$H_{i, \text { irr }}$ & $\begin{array}{c}\text { Contribution of the irradiation to } H \text { transported by the } i \text { th } \\
\text { thermal band }\end{array}$ & Eq. (99) & $\mathrm{W} / \mathrm{m}^{2}$ \\
\hline$H_{i, \text { int }}$ & $\begin{array}{c}\text { Contribution of the internal luminosity to } H \text { transported by the } \\
i \text { th thermal band }\end{array}$ & Eq. (99) & $\mathrm{W} / \mathrm{m}^{2}$ \\
\hline$T_{\text {eff }}$ & Effective temperature of the planet & Sect. 2.1 & $\mathrm{~K}$ \\
\hline$T_{\text {eff, } \mu_{*}}$ & Effective temperature of the modelled atmosphere & Sect. 2.1 & $\mathrm{~K}$ \\
\hline$T_{\text {skin }}$ & Temperature at optical depth of zero. & $\begin{array}{c}- \\
-\end{array}$ & K \\
\hline$T_{\text {deep }}$ & Temperature at large optical depth. & Eq. (109) & $\mathrm{K}$ \\
\hline$f$ & Parameter used to calculate dayside and planetary averages & Sect. 3.7 & - \\
\hline
\end{tabular}

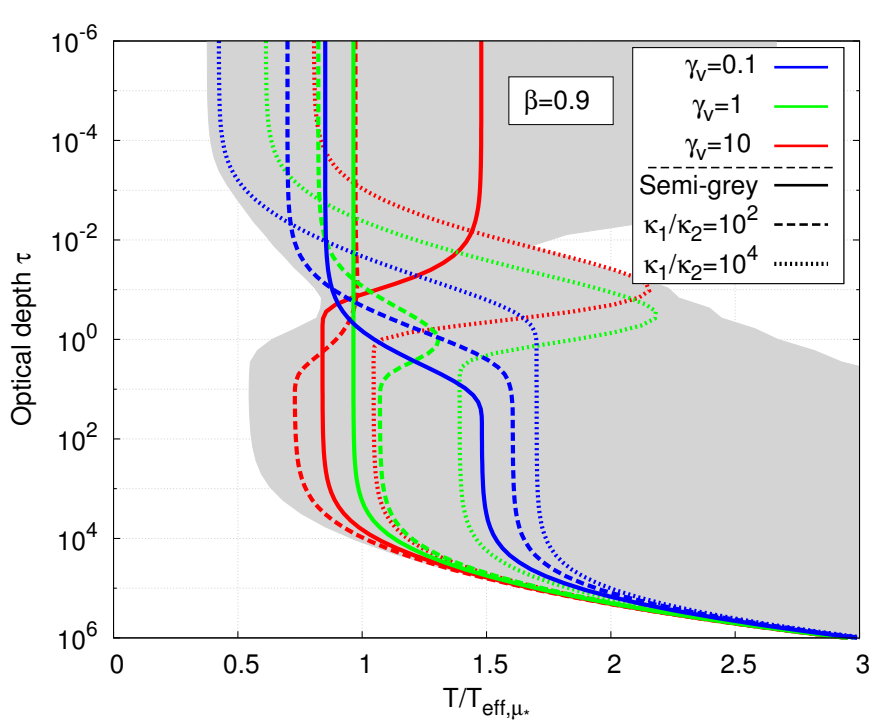

Fig. 14. Pressure/temperature profiles for an irradiated planet $\left(T_{\mathrm{int}}=\right.$ $T_{\text {irr }} / 10$ and $\mu_{*}=1 / \sqrt{3}$ ). The shaded area shows the full range of parameters $10^{-3}<1-\beta<10^{-1}, 1<R<10^{4}$ and $0.01<\gamma_{\mathrm{v}}<100$. The lines are profiles obtained for $\beta=0.9$; for $R=1$ (plain lines), $R=100$ (dashed lines), and $R=10^{4}$ (dotted lines); and for $\gamma_{\mathrm{v}}=0.1$ (blue), $\gamma_{\mathrm{v}}=1$ (green), and $\gamma_{\mathrm{v}}=10$ (red).

Rosseland mean opacities, the ones obtained from an opacity table for example. However, it cannot account for both realistic Rosseland mean and Planck mean opacities. Their ratio, $\gamma_{\mathrm{P}}$ and the width of the lines, $\beta$, must be held constant through the atmosphere. Although the model is limited to two thermal opacity bands, it can take into account any number of visible opacity bands, each band adding two new parameters, the strength of the band $\gamma_{\mathrm{v} i}$ and its width $\beta_{\mathrm{v} i}$

Our model solves the inability of previous analytical models to reach temperatures as cold as predicted by the numerical calculations. For opacities dominated by strong and narrow lines $(\beta<0.1)$, non-grey opacities lead to a colder upper atmosphere, but converges toward the grey model at optical depth greater than $\tau_{\text {lim }}$ (see Fig. 8). For opacities dominated by wide lines, or molecular bands $(\beta \approx 0.5)$, non-grey opacities still allow the upper atmosphere to cool down more efficiently, but also inhibit the cooling of the deep atmosphere. In that case, a significant warming of the deep atmosphere can happen, down to optical depths much greater than $\tau_{\text {lim }}$. This planetary blanketing effect could contribute to the radius anomaly of hot Jupiters.

Temperature inversions that were not predicted by previous analytical models occur whenever $\gamma_{\mathrm{v}}^{*} \tau_{\text {lim }} \approx 1$ because of the interaction between the incoming stellar irradiation and the nongrey thermal opacities. These could have interesting observational consequences.

We show that the internal flux is always transported by the spectral channel of lowest opacity. Conversely, the absorbed irradiation flux is transported by the spectral channel of lowest opacity only when $\gamma_{\mathrm{v}}^{*} \tau_{\lim }<1$. For values of $\gamma_{\mathrm{v}}^{*}$ larger than $\tau_{\lim }^{-1}$, it is transported by the spectral channel of highest opacity.We provide simple analytical expressions for the outgoing thermal flux in the different spectral bands.

Finally, our model allows for a much greater range of temperature profiles than other analytical and semi-analytical solutions of the radiative transfer equations for irradiated atmospheres. We encourage the community to use it when fast calculations of atmospheric temperature profiles are needed. Given 
V. Parmentier and T. Guillot: A non-grey analytical model for irradiated atmospheres. I.

the apparent complexity of the solution, a code is available at the CDS or at ww. oca.eu/parmentier/nongrey.

Acknowledgements. This work was performed in part thanks to a joint Fulbright Fellowship to V.P. and T.G. The whole project would not have been possible without the help and support of Douglas Lin. We also acknowledge Jonathan Fortney and Mark Marley for many useful discussions, and the University of California Santa Cruz for hosting us while this work was carried out.

\section{References}

Avrett, E. H., \& Krook, M. 1963, ApJ, 137, 874

Budaj, J., Hubeny, I., \& Burrows, A. 2012, A\&A, 537, A115

Burrows, A., Marley, M., Hubbard, W. B., et al. 1997, ApJ, 491, 856

Burrows, A., Hubeny, I., Budaj, J., Knutson, H. A., \& Charbonneau, D. 2007, ApJ, 668, L171

Chandrasekhar, S. 1935, MNRAS, 96, 21

Chandrasekhar, S. 1960, Radiative transfer (New york: Dover Publications)

Chevallier, L., Pelkowski, J., \& Rutily, B. 2007, J. Quant. Spectr. Radiat. Transf., 104,357

Eddington, A. S. 1916, MNRAS, 77, 16

Fortney, J. J., Lodders, K., Marley, M. S., \& Freedman, R. S. 2008, ApJ, 678, 1419

Guillot, T. 2010, A\&A, 520, A27

Guillot, T., \& Havel, M. 2011, A\&A, 527, A20

Guillot, T., \& Showman, A. P. 2002, A\&A, 385, 156

Hansen, B. M. S. 2008, ApJS, 179, 484

Heng, K., Menou, K., \& Phillipps, P. J. 2011, MNRAS, 413, 2380

Heng, K., Hayek, W., Pont, F., \& Sing, D. K. 2012, MNRAS, 420, 20

Hubeny, I., \& Lanz, T. 1995, ApJ, 439, 875

Hubeny, I., Burrows, A., \& Sudarsky, D. 2003, ApJ, 594, 1011
King, I. J. I. F. 1955, ApJ, 121, 711

King, J. I. F. 1956, ApJ, 124, 272

Krook, M. 1963, ApJ, 137, 863

Laughlin, G., Crismani, M., \& Adams, F. C. 2011, ApJ, 729, L7

Line, M. R., Zhang, X., Vasisht, G., et al. 2012, ApJ, 749, 93

Matsui, T., \& Abe, Y. 1986, Nature, 322, 526

Meador, W. E., \& Weaver, W. R. 1980, Journal of Atmospheric Sciences, 37, 630

Mihalas, D. 1978, Stellar atmospheres, 2nd edn. (W.H. Freeman and Co.)

Mihalas, D., \& Mihalas, B. W. 1984, Foundations of radiation hydrodynamics (New york: Oxford University Press)

Miller-Ricci, E., \& Fortney, J. J. 2010, ApJ, 716, L74

Milne, E. A. 1921, MNRAS, 81, 510

Mordasini, C., Alibert, Y., Georgy, C., et al. 2012a, A\&A, 547, A112

Mordasini, C., Alibert, Y., Klahr, H., \& Henning, T. 2012b, A\&A, 547, A111

Münch, G. 1946, ApJ, 104, 87

Parmentier, V., \& Guillot, T. 2011, in EPSC-DPS Joint Meeting 2011, 1367

Pierrehumbert, R. T. 2010, Principles of Planetary Climate (Cambridge: University Press)

Pujol, T., \& North, G. R. 2003, Tellus A, 55, 328

Rauscher, E., \& Menou, K. 2013, ApJ, 764, 103

Rauscher, E., \& Showman, A. P. 2013, ApJ, submitted [arXiv: 1309.7052]

Robinson, T. D., \& Catling, D. C. 2012, ApJ, 757, 104

Rutily, B., Chevallier, L., Pelkowski, J., \& Bergeat, J. 2008, J. Quant. Spectr. Radiat. Transf., 109, 28

Saumon, D., Hubbard, W. B., Burrows, A., et al. 1996, ApJ, 460, 993

Seager, S., \& Deming, D. 2010, ARA\&A, 48, 631

Shaviv, N. J., Shaviv, G., \& Wehrse, R. 2011, Icarus, 216, 403

Showman, A. P., Fortney, J. J., Lian, Y., et al. 2009, ApJ, 699, 564

Spiegel, D. S., \& Burrows, A. 2013, ApJ, 772, 76

Unno, W., \& Yamashita, Y. 1960, PASJ, 12, 157

Weaver, C. P., \& Ramanathan, V. 1995, J. Geophys. Res., 100, 11585 